\title{
A Method for Estimation of Extreme Values of Wind Pressure on Buildings Based on the Generalized Extreme-Value Theory
}

\author{
Yong Quan, Fei Wang, and Ming Gu \\ State Key Laboratory for Disaster Deduction in Civil Engineering, Tongji University, Shanghai 200092, China \\ Correspondence should be addressed to Ming Gu; minggu@tongji.edu.cn
}

Received 22 August 2013; Revised 12 December 2013; Accepted 15 December 2013; Published 21 January 2014

Academic Editor: Usik Lee

Copyright (C) 2014 Yong Quan et al. This is an open access article distributed under the Creative Commons Attribution License, which permits unrestricted use, distribution, and reproduction in any medium, provided the original work is properly cited.

\begin{abstract}
By analysis of statistical characteristics and probability density distribution of extreme values of wind pressures on the surfaces of a typical low-rise building model and a typical high-rise building model, characteristics of the commonly used methods for estimating the extreme-values of wind pressure are discussed. The relationship between the parameters of the extreme value distribution of wind pressure and its observation length is then deduced based on the generalized extreme value theory and the independence of the observed extreme values. A new method for estimating the extreme values is developed by dividing the time history sample of the wind pressure into several subsamples. The extreme values of the wind pressure coefficients calculated with the present method and those with the commonly used methods are compared and the results indicate that the present method can estimate the extreme values of non-Gaussian wind pressure more accurately than the commonly used ones.
\end{abstract}

\section{Introduction}

Wind pressure on building surfaces is a random process, and its probabilistic and statistical characteristics are some of the key points to wind engineering. In the 1960s, Davenport [1] introduced statistical concepts into wind engineering and assumed that the wind speed, wind angle, and wind pressure coefficients all satisfy the Gaussian distribution. Until now, many studies on analyzing and modeling of wind effects still accept that the random processes involved in wind pressure assume a Gaussian process. This concept is mainly used to facilitate the analysis of wind pressures; many Gaussian processes are known to exist. This assumption is effective when the overall effect of random wind pressure field on an area is considered.

Peterka and Cermak [2] and Kareem [3] indicated that, in an area where the mean wind pressure coefficients are lower than -0.25 , the wind pressure is generally skewed. They found many spikes in the wind pressure history. These spikes are six times the root-mean-square value from the mean values. The probability of their occurrence is much greater than that predicted by the Gaussian distribution. After many studies on the wind pressure on buildings, Stathopoulos [4] also indicated the considerable skewness of wind pressure data in certain areas. Tieleman and Reinhold [5] and Holmes and Best [6] also reached a similar conclusion. As the building is within the lower part of the atmospheric boundary layer, which experiences high turbulence, and surrounding obstacles are present, the windward wall of the building suffers from a non-Gaussian wind pressure. The nonGaussian characteristic of the fluctuating wind may result in its amplified effects. Holmes [7] and Kawai [8] estimated the probability density function of the wind pressure using the quasi-steady and strip theories. They found consistency between the estimated probability density function and the probability density function of the measured data in the area of the air flow reattachment. However, due to the additional factors arising from the interaction of the structure and wind with different turbulence intensities, the quasi-steady theory cannot be used to estimate the probability density function of the wind pressure in the area of air flow separation. Letchford et al. [9] substantiated this view using measured data. Thomas et al. [10] maintained that the quasi-steady theory cannot describe the spectrum of the wind pressure in the air flow 
separation area even if it contains the squared term of the fluctuating wind velocity. Tieleman et al. [11] arrived at a similar conclusion, using measured data.

Estimating the extreme value of non-Gaussian wind pressure is another important problem in wind engineering. Davenport [12] estimated the extreme values of wind pressures by summing their mean values and standard deviation multiplied by a peak factor obtained with the level-cross method, in which the fluctuating wind pressures were assumed to be Gaussian. This method is known as the peak factor or Davenport method. However, local wind pressures against the claddings, or construction components (especially under high turbulence), and in the separation area severely deviate from the Gaussian distribution. In this case, extreme values obtained from the Davenport method are usually smaller than actual values; designs based on such deviating results are unsafe $[13,14]$.

To solve this problem, Kareem and Zhao [15] transformed the Gaussian random variable to a Hermite polynomial of a non-Gaussian random variable with their high-order moments (skewness coefficients and kurtosis coefficients). This extended the applicability of the Davenport method beyond Gaussian processes. Cartwright and Longuet-Higgins [16] introduced the concept of bandwidth parameter to solve the water wave problem. Pillai and Tamura [17] discussed the peak factor for non-Gaussian and narrow/broad band processes based on the achievements mentioned above. On the other hand, Kwon and Kareem [18] improved their previous method on some shortcomings and proposed a more accurate method for estimating extreme values (KwonKareem method). Sadek and Simiu [19] applied the translation process approach proposed by Grigoriu [20] to construct the non-Gaussian process map from the Gaussian process, although they estimated the extreme values of wind pressure by the Davenport method. Their method is also known as the Sadek-Simiu method. Ge [21] showed that the Sadek-Simiu method is more reliable than the other methods, based on data from wind tunnel tests.

Kasperski [22] studied the probability distribution of extreme values, using more than 3000 samples from wind tunnel tests. The results of this study show that the probability distribution of extreme values of wind pressure can be described accurately by the extreme distribution types I and III. Holmes and Cochran [23, 24] fitted the extreme values of thousands of tested wind pressure with the extreme distribution type I and the generalized extreme value (GEV) distribution, which is the preferred system for estimating the extreme values because the extreme values of these samples are stated directly. Although this method is widely used in the estimation of the extreme values of wind velocity and flood water levels, it is not suitable for estimating the extreme wind pressure coefficients on the surface of a structure in practical engineering because it requires a large number of samples. Obtaining this quantity of samples from wind tunnel tests is not economical. A method to estimate the extreme values using only one sample with an appropriate length is very practical. Peterka [25] designed such a method by selecting several extreme values in a sample, fitting these extreme values with extreme distribution type I, and estimating the extreme values. Lieblein [26] proposed that the two parameters of extreme distribution type I can be calculated by using the BLUE method accurately (BLUE method). However, the probability distribution of the extreme values of wind pressures does not always satisfy the assumption of extreme distribution type I.

In this work, the surface wind pressure data on a typical low-rise building model and a typical high-rise building model, which were obtained from wind tunnel tests in simulated wind field, were analyzed. The statistical characteristics of the data were examined. The relationship between the parameters of the extreme value distribution of the wind pressure and its observation length was deduced based on the GEV theory. A new method of estimating extreme values was developed by dividing a sample of the wind pressure time history into several subsamples.

\section{Brief Introduction of Wind Tunnel Tests on a Low-Rise Building Model and a High-Rise Building Model}

A large number of wind tunnel tests on low-rise and high-rise buildings have been performed on this subject. The surface wind pressure data from a wind tunnel test on one of the low-rise building models in a simulated suburban wind field was chosen. The test model, the definition of the wind angle, and the arrangement of the wind pressure taps are shown in Figure 1. Measurements were obtained from a 1/100 scale model. The roof slope, $\beta$, is $21.8^{\circ}$. The ratio of the height $H$, width $B$, and depth $D$ is $3: 4: 6$. The mean velocity at the height of $10 \mathrm{~cm}$ during the test was about $10 \mathrm{~m} / \mathrm{s}$ with a wind velocity scale $1 / 3$. The mean-velocity profile could also be fitted with a power law with an exponent of 0.2 . The simulated turbulent intensity of the approaching wind at the roof height is about 0.25 . The frequency response of the pressure measurement system was $500 \mathrm{~Hz} .15$ repeated $18 \mathrm{~s}$ time series samples of pressure coefficients were obtained.

Figure 2 shows the test model, the definition of the wind angle, and the arrangement of the wind pressure taps of the high-rise building. The scale ratios of length, wind velocity and time in this test were $1 / 300,1 / 3$, and $1 / 100$, respectively. The sampling frequency and sampling time of the wind pressure were $312.5 \mathrm{~Hz}$ and $42 \mathrm{~s}$, corresponding to $3.13 \mathrm{~Hz}$ and 70 minutes in full scale, respectively. The mean velocity at the top of the building model during the test was about $8.1 \mathrm{~m} / \mathrm{s}$, where the simulated turbulent intensity is about 0.10 .400 time history samples of wind pressures were obtained.

\section{Probability and Statistics Characteristics of Wind Pressures on the Surfaces of a Building}

Methods for estimating the extreme values of wind pressures are always based on a certain probability or statistics assumption. In this section, the theoretical basis of those commonly used methods for estimating the extreme values of wind pressure is discussed through making an assay of the probability and statistics characteristics of the wind pressures 


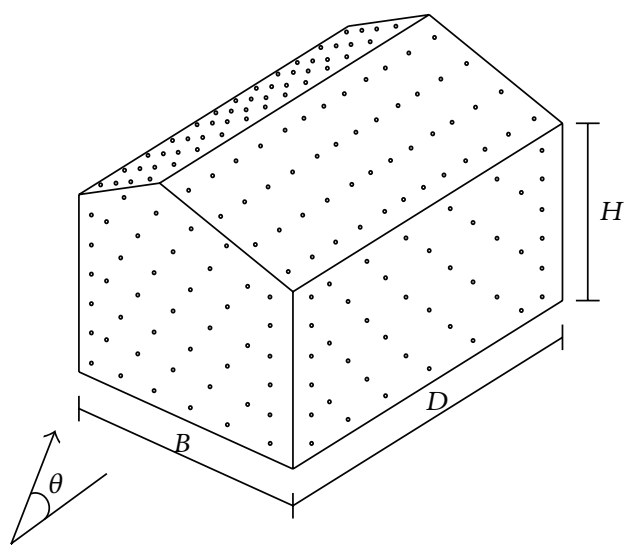

\begin{tabular}{llllllllllll}
\hline 0 & & 0 & & 0 & & 0 & & 0 & & 0 & 0 \\
0 & 0 & & 0 & & 0 & & 0 & & 0 & & 0 \\
0 & & 0 & & 0 & & 0 & & 0 & & 0 & 0 \\
0 & 0 & & 0 & & 0 & & 0 & & 0 & & 0 \\
0 & & 0 & & 0 & & 0 & & 0 & & 0 & 0 \\
0 & 0 & & 0 & & 0 & & 0 & & 0 & & 0 \\
\hline
\end{tabular}

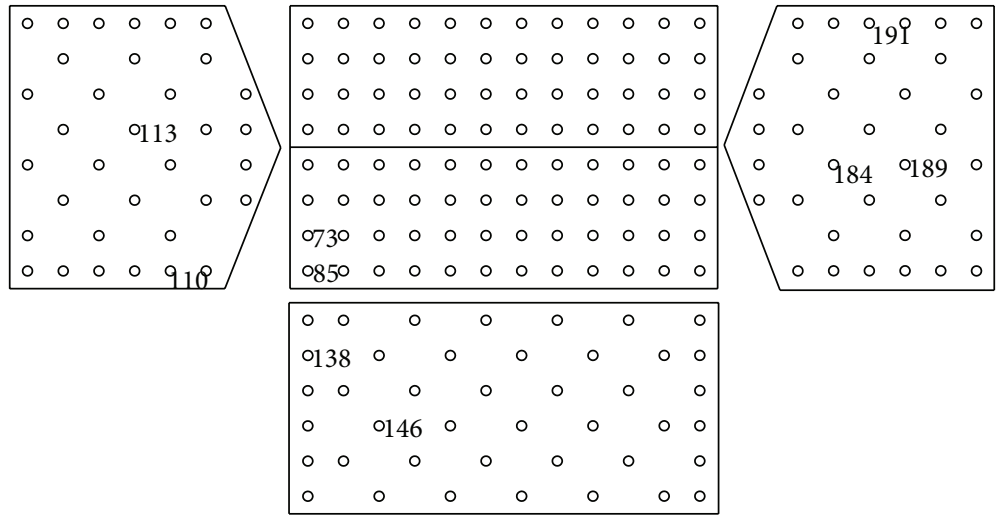

Figure 1: The test model, the definition of the wind angle, and the arrangement of the test taps of the low-rise building model.

on the surfaces of the low-rise building as well as the high-rise building.

3.1. High-Order Moment Analysis. Kumar and Stathopoulos [27] indicated that the standard Gaussian process should have a skewness range of $-0.5 \sim 0.5$ and a kurtosis range of 2.5 3.5. To describe intuitively the non-Gaussian characteristic of the surface wind pressure on the low-rise building model, the skewness and the kurtosis in test cases of 0,45 , and $90^{\circ}$ wind angles were calculated; their contour figures are depicted (Figures 3 and 4).

The skewness of the wind pressure coefficients on the windward wall (Figure 3 ) is generally positive, up to about 1.0. They are negative at the other walls and on the roof. The negative skewness of the wind pressure coefficients at side walls and on the roof is significant, especially those in the oblique directions. The skewness in the windward roof corner area is particularly significant and reaches -3.0 . However, the negative skewness is relatively small on the downwind roof, the downwind side, and the leeward walls. From the test data, the skewness of the whole roof and the walls highly deviates from the skewness range of the standard Gaussian processes. Most of the kurtoses of the wind pressure coefficients on the building surfaces are more than 4.0 (Figure 4), and the kurtoses at the downwind side walls for the $0^{\circ}$ wind angle and at the windward roof corner for the $45^{\circ}$ wind angle are significant (maximum value of 25 ). These characteristics seriously deviate from standard Gaussian features.

The non-Gaussian characteristics of the surface wind pressure are also ubiquitous on the high-rise building model. The skewness and the kurtosis in test cases of 0,45 , and $90^{\circ}$ wind angles were calculated and their contour figures are described in Figures 5 and 6. It can be seen from the figure that the skewness at the side and leeward walls is negative.
In most area, the skewness is less than -0.5 and the kurtoses are more than 4.5 , especially in the middle area at the bottom of the model, the skewness even up to -1.5 and the kurtosis up to 7.5. And it seriously deviates from the scope of the standard Gaussian process. What is more, it is also obvious that the non-Gaussian characteristics in the bottom area of the leeward walls in test case of $45^{\circ}$ wind angle and the leeward wall in test case of $90^{\circ}$ wind angle are widespread and strong.

Since the wind pressure coefficients of the building cannot satisfy the assumption of a Gaussian process, the Davenport method cannot reliably estimate extreme values of the wind pressure.

Kareem and Zhao transformed the Gaussian random variable to a Hermite polynomial of a non-Gaussian random variable with the high-order moments by using the following expression:

$$
x=\alpha\left\{u+h_{3}\left(u^{2}-1\right)+h_{4}\left(u^{3}-2 u\right)\right\},
$$

where

$$
\begin{gathered}
\alpha=\left(1+2 h_{3}^{2}+6 h_{4}^{2}\right)^{-1 / 2}, \\
h_{3}=\frac{\gamma_{3}}{4+2 \sqrt{1+1.5 \gamma_{4}}}, \\
h_{4}=\frac{\sqrt{1+1.5 \gamma_{4}}-1}{18},
\end{gathered}
$$

where $\gamma_{3}$ and $\gamma_{4}$ are the skewness and excess kurtosis of the wind pressure time history and $u$ represents a standard Gaussian process.

Kwon and Kareem improved the calculating method about the coefficients $h_{3}$ and $h_{4}$, intending to make (1) 


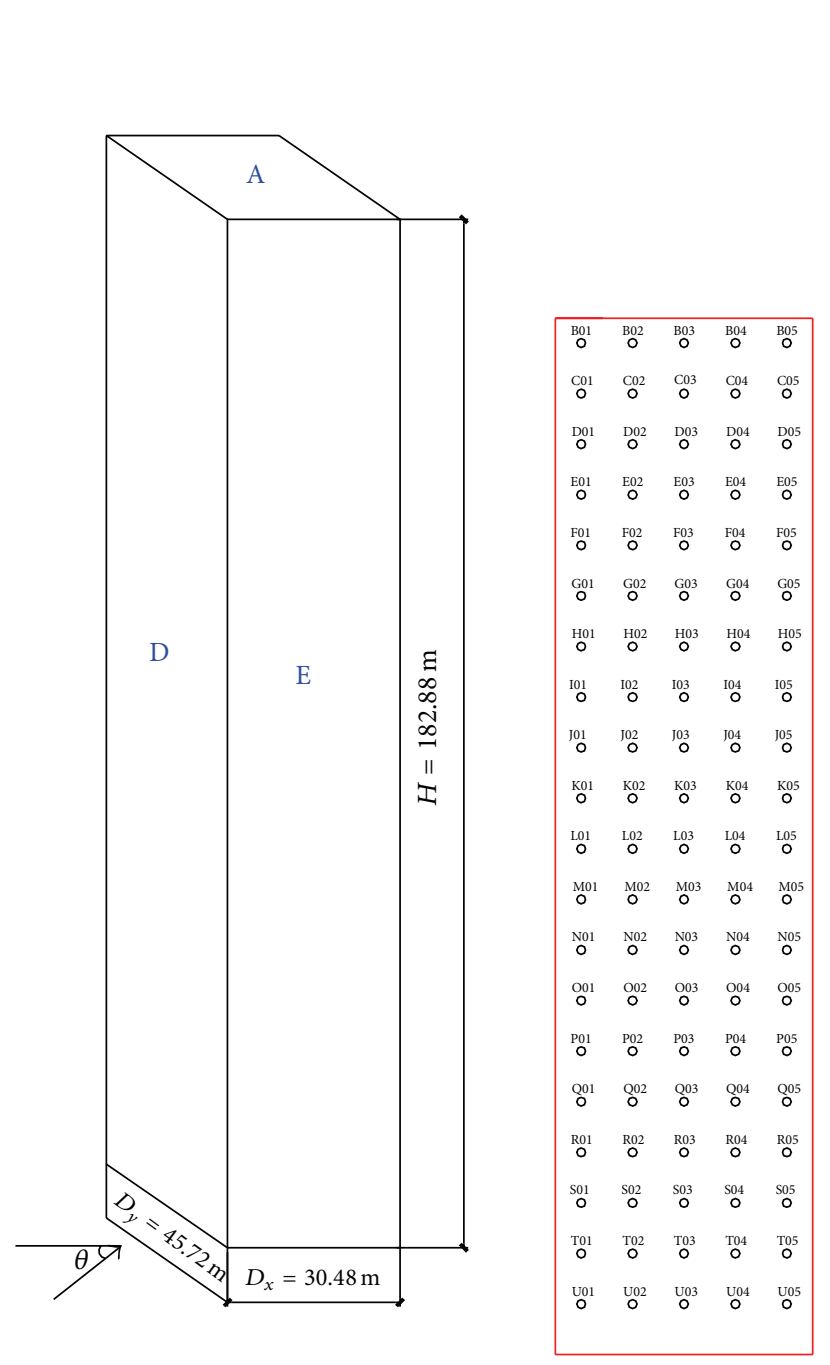

Taps on face B

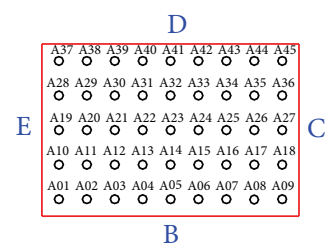

Taps on face A

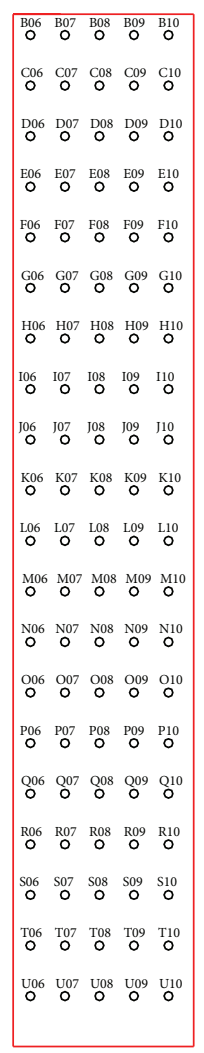

Taps on face $\mathrm{C}$

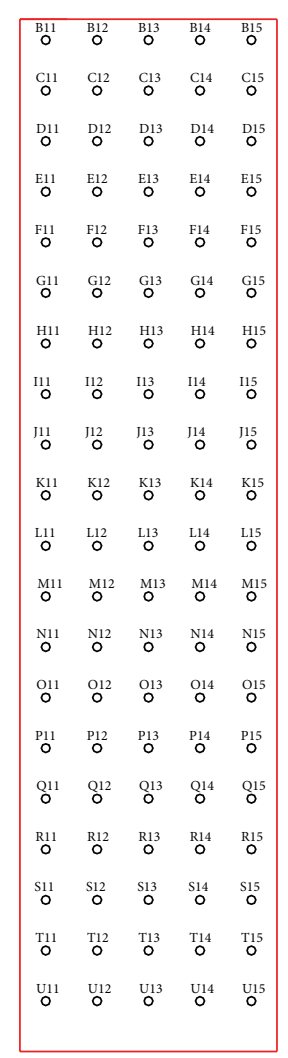

Taps on face D

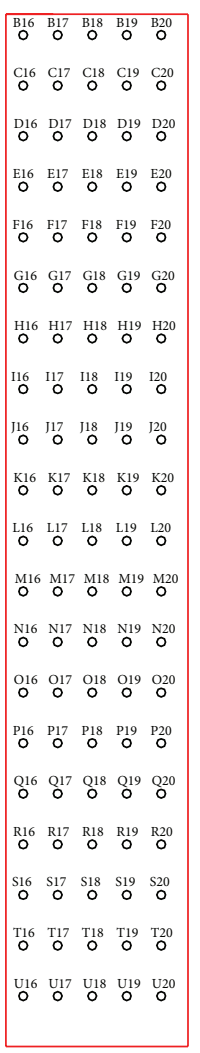

Taps on face E

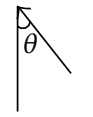

FIgURE 2: The test model, the definition of the wind angle, and the arrangement of the test taps of the high-rise building model.

still applicable for the strong non-Gaussian process through solving the following equations:

$$
\begin{gathered}
\gamma_{3}=\alpha^{3}\left(8 h_{3}^{3}+108 h_{3} h_{4}^{2}+36 h_{3} h_{4}+6 h_{3}\right), \\
\gamma_{4}+3=\alpha^{4}\left(60 h_{3}^{4}+3348 h_{4}^{4}+2232 h_{3}^{2} h_{4}^{2}\right. \\
+60 h_{3}^{2}+252 h_{4}^{2}+1296 h_{4}^{3} \\
\left.+576 h_{3}^{2} h_{4}+24 h_{4}+3\right) .
\end{gathered}
$$

However, in theory, it is unavoidable to encounter the truncation error while using the first four-order statistics to establish the polynomial to fit the actual probability density distribution of wind pressure. In order to describe the error of this fitting process, the standardization of the non-Gaussian wind pressure on surfaces of the building was done in the first place. And then, an inverse transformation was taken by using (1) so that the standardized non-Gaussian process was mapped into the corresponding Gaussian time history. Through comparing the mapping results with the standard Gaussian process, it is clear to explain whether the proposed polynomial can fit the non-Gaussian wind pressure well, which is also the keystone of the Kwon-Kareem method.

The comparative results of representative test taps on surfaces of the high-rise building model in test cases of 0 and $45^{\circ}$ wind angles were considered (Figure 7). It can be seen from the figure that the proposed polynomial can give a good mapping results when the non-Gaussianity of the wind pressure is slight. However, if a strong non-Gaussian process is encountered, its mapping result will show a significant difference on both tails of the probability distribution. For these test taps, (1) cannot provide satisfactory fitting results 


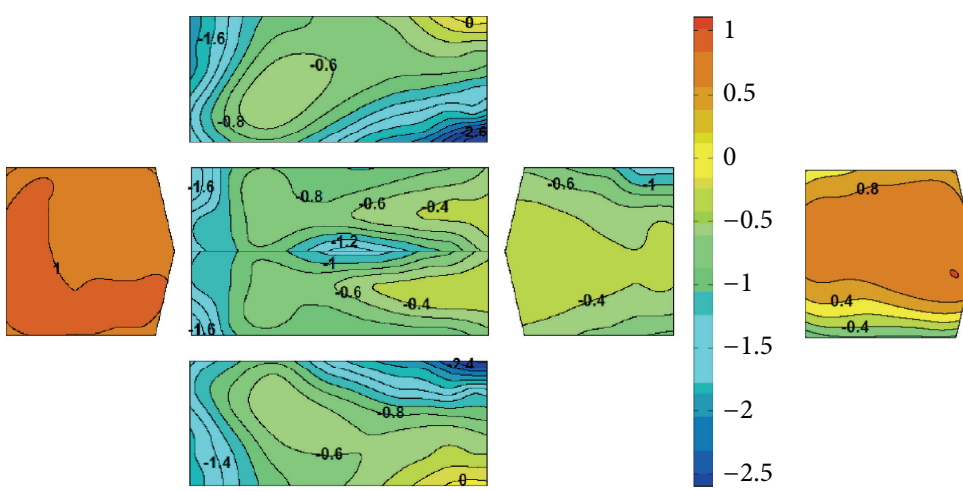

(a) $0^{\circ}$ wind angle
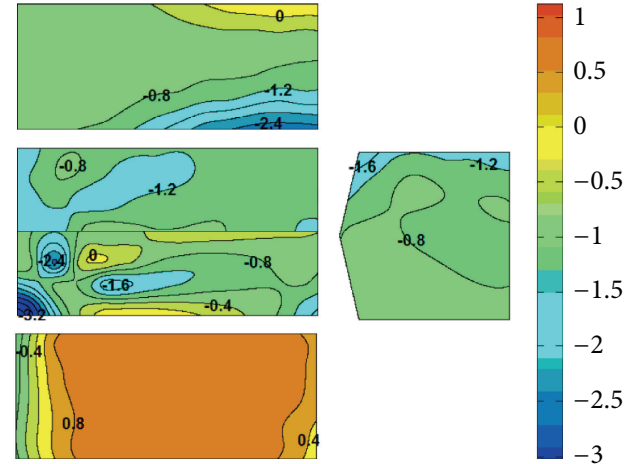

(b) $45^{\circ}$ wind angle

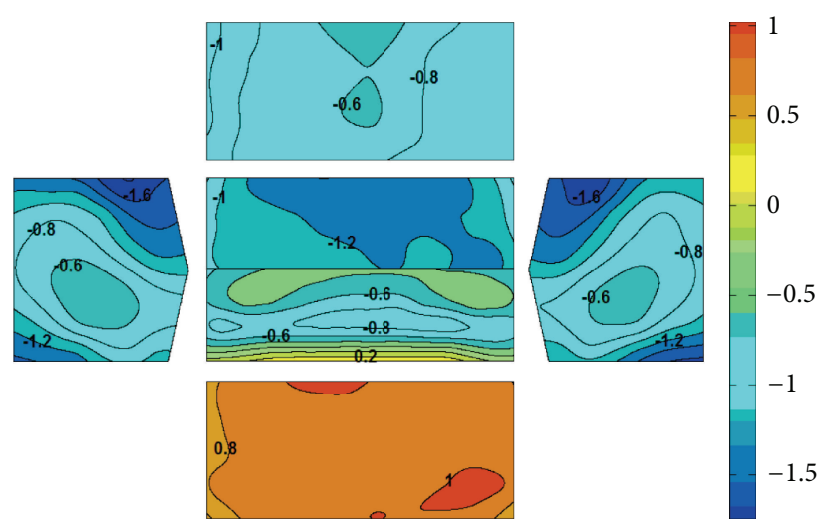

(c) $90^{\circ}$ wind angle

FIGURE 3: Skewness contours of the wind pressure coefficients at the model surface at different wind angles.

on their probability density distribution. It is a limitation for us to adopt the Kwon-Kareem method to estimate the extreme values of wind pressure. The comparative results of representative test taps on surfaces of the low-rise building model also confirm this conclusion.

3.2. Probability Density Distribution Analysis of Wind Pressure. The probability density distribution functions of the wind pressure coefficients of representative test taps were analyzed (Figures 8 and 9). The results of fitting with the Gaussian, GEV, lognormal, and gamma distributions with three parameters are illustrated in the figure. The maximum likelihood method was used to estimate parameters of the GEV distribution, whereas the moment estimation method was used to estimate those of the lognormal and gamma distributions. However, as the three parameters of the lognormal distribution cannot be expressed explicitly, the reduced form of Chen et al [28] was adopted. In addition, the expression proposed by Tieleman and Hajj [29] was used.

As seen from the figures, the wind pressure coefficients of typical areas in the test taps seriously deviate from the Gaussian distribution. The lognormal, gamma, and GEV distributions provide a better fit than the Gaussian distribution does. However, the fitting quality of the distribution patterns varies. The patterns generally do not show a good fit with the tail of the probability distribution function for the wind pressure, which is the key to estimating extreme values. The surface wind pressure at various surfaces of the building has poor fit with any pattern of the probability distribution function, and the wind pressure coefficients of the different test taps are not consistent, which are similar to previous observations [30]. Sadek and Simiu [19] have drawn a conclusion that the gamma and Gaussian distributions can describe the wind pressures on the surface of low-rise buildings well. The time series with positive skewness, the gamma distribution is appropriate for estimating the positive extreme values, while a normal distribution is appropriate for estimating the negative extreme values. For those records with negative skewness, the same conclusion holds after multiplication of the original time series by -1 . However, as seen from the figure, for those taps with strong nonGaussianity, both tails of the probability distribution cannot be fitted satisfactorily by these two patterns, with great errors existing. According to the maximum domain of attraction theory, the gamma and Gaussian distribution both belong to the maximum attraction domain of extreme value type I distribution. And yet, the probability density distribution of the extreme values of wind pressure on the surfaces of buildings always does not comply with the extreme value type I distribution. The Sadek-Simiu method, which makes an attempt to use the gamma and Gaussian distribution to 


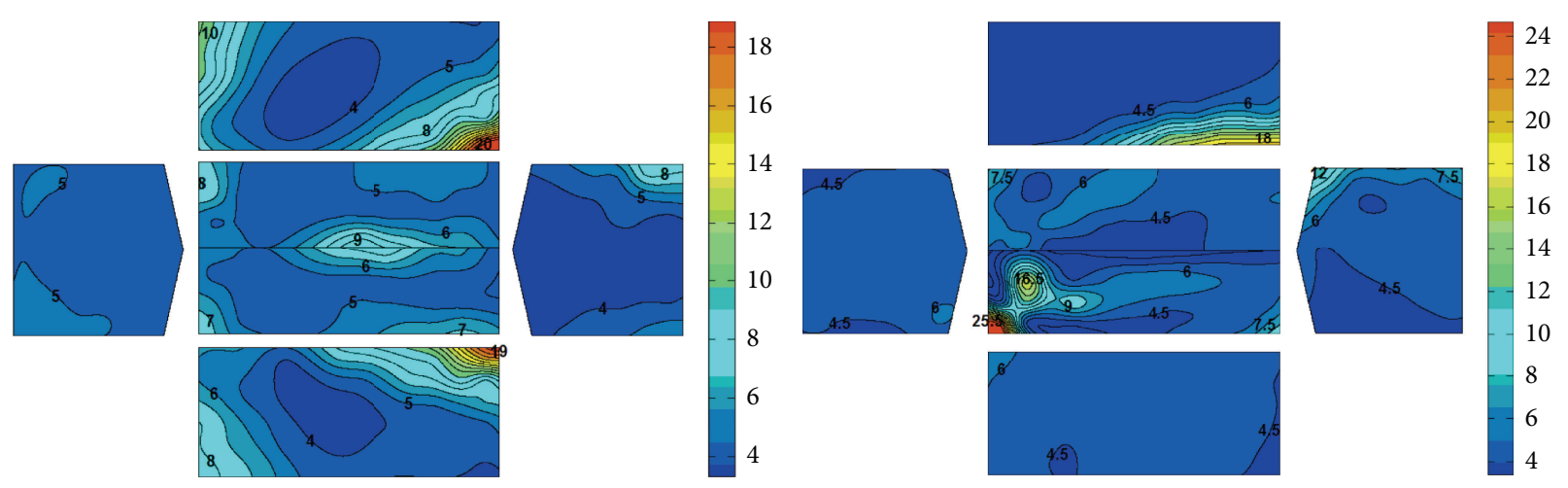

(a) $0^{\circ}$ wind angle

(b) $45^{\circ}$ wind angle

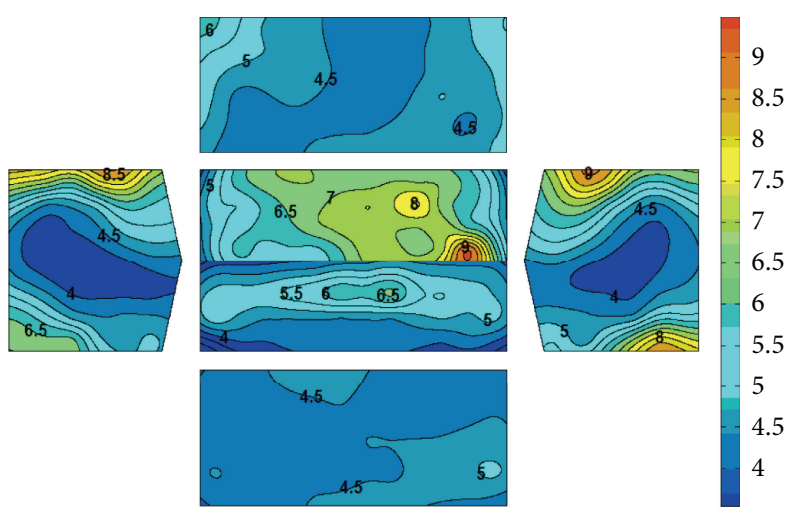

(c) $90^{\circ}$ wind angle

FIGURE 4: Kurtosis contours of the wind pressure coefficients at the low-rise building model surface at different wind angles.

describe the probability distribution of the parent sample so as to estimate extreme values, clearly suffers from this problem.

In sum, the probability distribution of the non-Gaussian wind pressure time history on the surfaces of the buildings cannot be fitted by one certain existing mathematical model well. Both the Sadek-Simiu method based on the commonly used probability distribution functions and Kwon-Kareem method relied on the polynomial established by the first fourorder statistics still have some problems while they are used to describe the probability distribution of wind pressure on the surfaces of buildings. Moreover, these two estimating methods are based on the level-crossing rate theory. And the raw non-Gaussian time history was used to replace mapping result while calculating the level-crossing rate because the mapping process approach cannot obtain the standard Gaussian process. It might bring great errors, especially for the Kwon-Kareem method. However, if a method based on extreme-value theory was taken to estimate the extreme values of wind pressures, the problems faced by the methods mentioned above could be avoided effectively.

3.3. Probability Distribution Analysis of Extreme Values. According to the theory raised by Fisher and Tippett [31], for independent and identically distributed sequence of random variables, if the number of samples is sufficient, the probability distribution of their extreme values will obey the generalized extreme value distribution. Through an analysis of large number of wind pressure samples, Kasperski [22] held an opinion that the extreme values of wind pressure on surfaces of buildings generally comply with the extreme value types I and III. And in this paper, 2800 time history samples of the high-rise building model were obtained so as to make an assay of the probability distribution of the wind pressure (Figure 10). The analyzing result indicates that the wind pressure extreme values on the high-rise building adhere to the extreme value types I and III, which is in accord with the Kasperski's conclusion. The shape parameter of the probability distribution becomes more and more stable with the increase of the number of samples. In view of the research carried out by Holmes and Cochran [24] which owns the opinion that 4000-5000 independent time series might be required to identify the finite tail of the extreme value type III, the number of independent extreme values here is absolutely insufficient. And as seen from the fitting results in Figure 9, it is also obvious that the fitting results are not satisfactory enough in the rear part even if 2800 independent time series were taken. Consequently, in engineering, a method to estimate the extreme values using only one sample with an appropriate length is very practical.

The probability distributions of the positive and negative extreme values of a wind pressure time history for different 


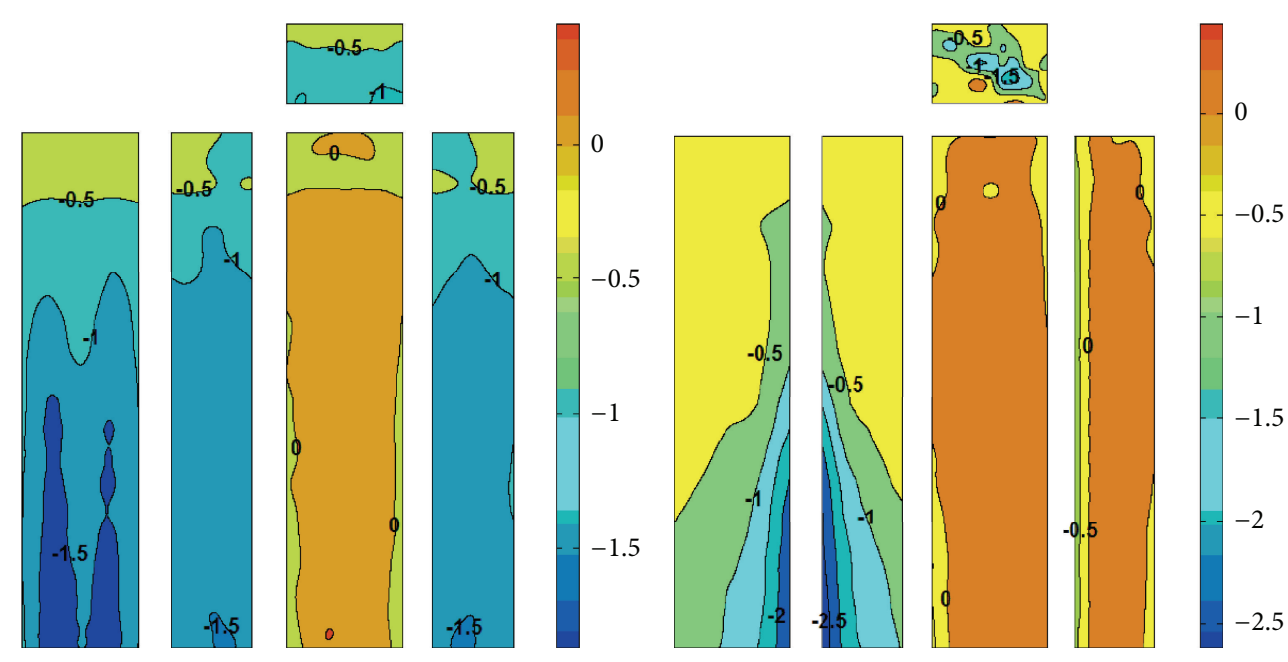

(a) $0^{\circ}$ wind angle

(b) $45^{\circ}$ wind angle

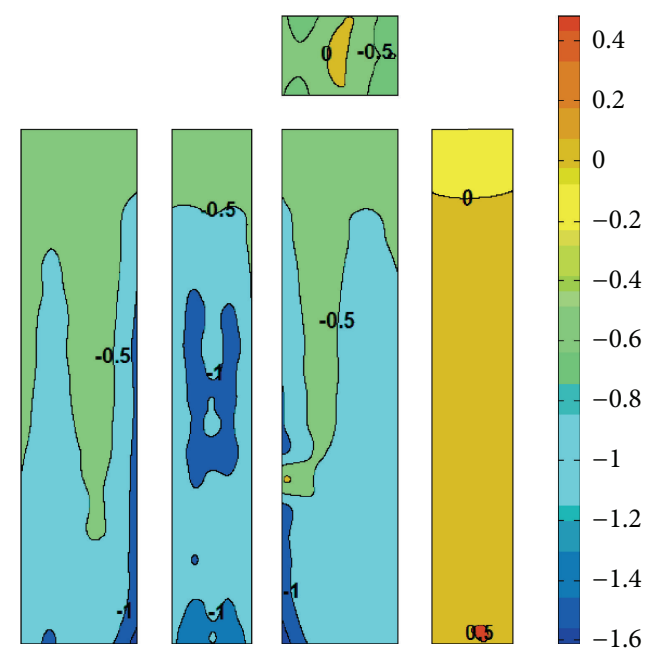

(c) $90^{\circ}$ wind angle

FigURE 5: Skewness contours of the wind pressure coefficients at the model surface at different wind angles.

wind angles at several test taps of low-rise building and highrise building are shown in Figures 11 and 12, separately. In the figure, results of fitting with the extreme value type I (Gumbel) and GEV distributions are also illustrated. The extreme values in the figure are those of the sub-samples obtained according to the optimal observation period (Section 4). The distributions of the extreme values of wind pressure coefficients do not always follow the Gumbel distribution. Additionally, although the number of independent extreme values from a single time history obtained from the subsamples here is far away from the requirement proposed by Holmes and Cochran, the application of the GEV distribution function could greatly improve the fit with the extreme value type I distribution. Therefore, the extreme estimation methods proposed by Peterka as well as Lieblein, which are both based on the extreme value type I distribution, still need further improvement.

What is more, the wind pressure extreme values on surfaces of buildings still comply with the extreme value types I and III while the extreme value type II seldom appears except those which are likely to comply with the extreme value type II distribution due to the error in the individual values. Statistical analyses show that these errors have a significant effect on the final estimate of the extreme values. Thus, results for fit with extreme value type I distribution were used to replace those for extreme value type II.

\section{Method for Estimating Extreme Values Based on the GEV Theory}

From the above analysis, the current methods of estimating wind pressure extreme values still have some inadequacies in their assumption of the wind pressure distribution. Hence, a new method of estimating extreme values is developed in the present study; it is based on the GEV theory, which is highly versatile. 


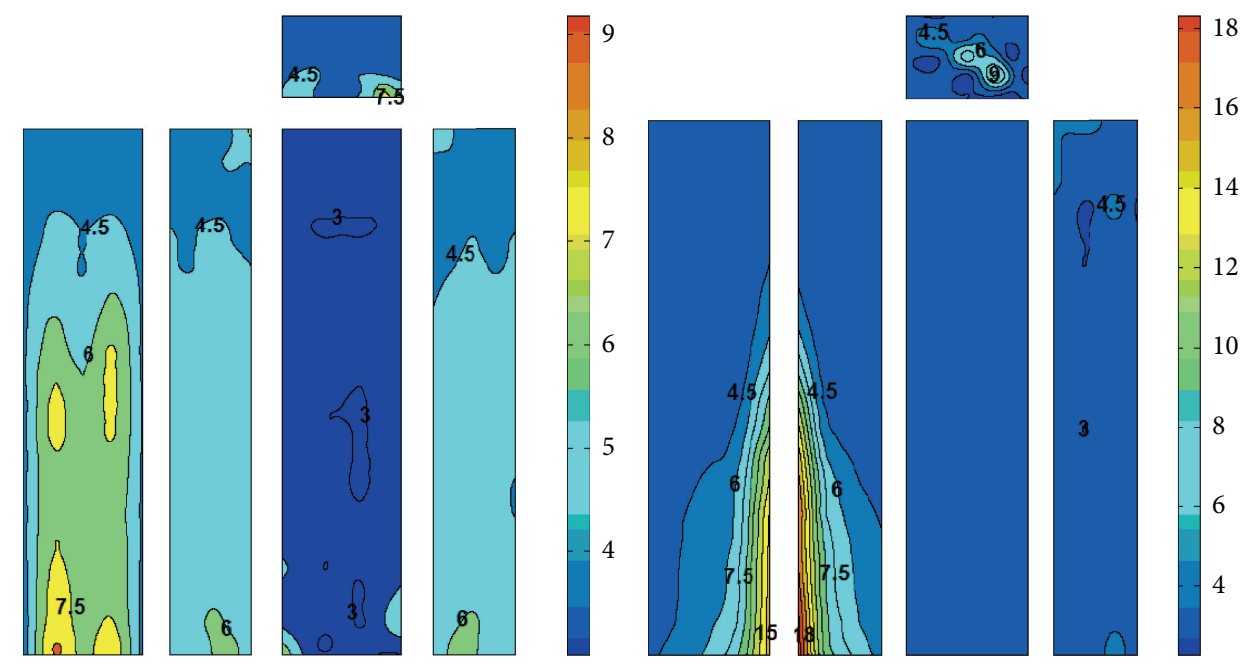

(a) $0^{\circ}$ wind angle

(b) $45^{\circ}$ wind angle

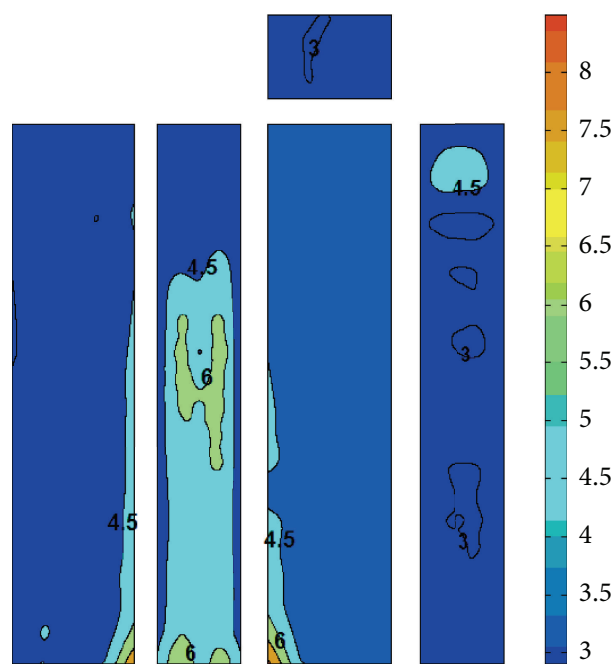

(c) $90^{\circ}$ wind angle

FIGURE 6: Kurtosis contours of the wind pressure coefficients at the low-rise building model surface at different wind angles.

4.1. The GEV Theory and the Reasoning Process for Prediction of Extreme Values. The classical extreme value theory considers that a large number of independently observed extreme values fit one of the three classes of distribution regardless of the probability distribution of the maternal sample [31]. In addition, these three classes of distribution can be combined into a single family of models having distribution functions of the form

$$
F\left(x_{e}\right)=\exp \left\{-\left[\frac{1-k\left(x_{e}-\mu\right)}{\sigma}\right]^{1 / k}\right\}
$$

Defined on the set $\left\{x_{e} \mid 1-k\left(x_{e}-\mu\right) / \sigma>0\right\}$, where $F\left(x_{e}\right)$ is the probability not greater than $x_{e}$ and $\mu, \sigma$, and $k$ are the location, scale, and shape parameters, respectively.
If $k$ is zero, then (4) is reduced to (5), which is the extreme value type I probability distribution function:

$$
F\left(x_{e}\right)=\exp \left\{-\exp \left[\frac{-\left(x_{e}-\mu\right)}{\sigma}\right]\right\}
$$

The probability intensity distribution function of the GEV distribution can be obtained by differentiating both sides of (4):

$$
\begin{aligned}
p_{X}\left(x_{e}\right)= & \frac{1}{\sigma} \exp \left\{-\left[\frac{1-k\left(x_{e}-\mu\right)}{\sigma}\right]^{1 / k}\right\} \\
& *\left[\frac{1-k\left(x_{e}-\mu\right)}{\sigma}\right]^{1 / k-1} .
\end{aligned}
$$




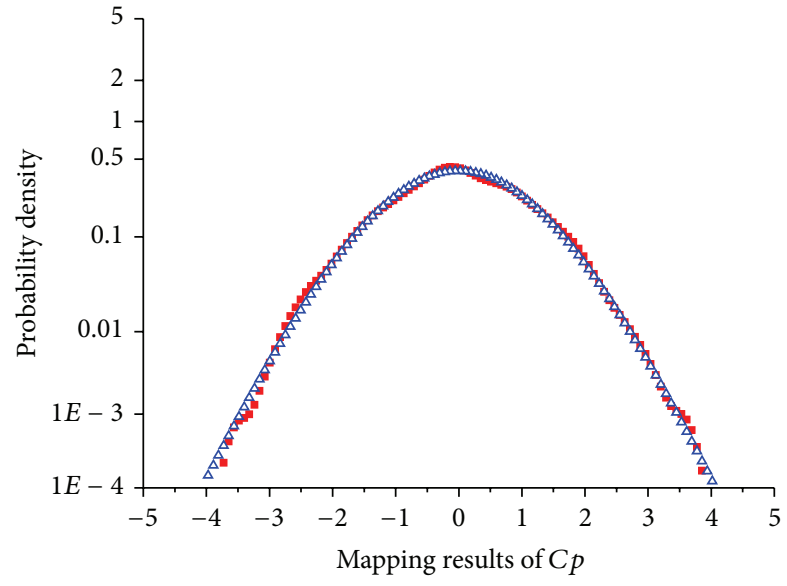

Wind pressure coefficients of tap $\mathrm{H} 13$ in wind angle 0 $\left(E_{C p}=0.87 ; s_{C p}=0.17 ; C_{s-C p}=0.15 ; C_{k-C p}=2.92\right)$

- Mapping results

$\Delta$ Standard gaussian process

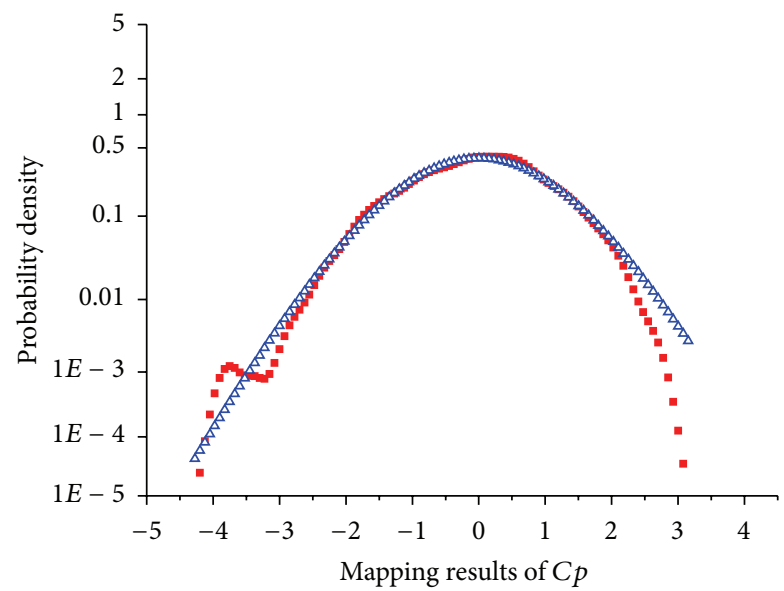

Wind pressure coefficients of tap M09 in wind angle 0

$\left(E_{C p}=-0.83 ; s_{C p}=0.33 ; C_{s-C p}=-1.54 ; C_{k-C p}=4.82\right)$

- Mapping results

$\Delta$ Standard gaussian process

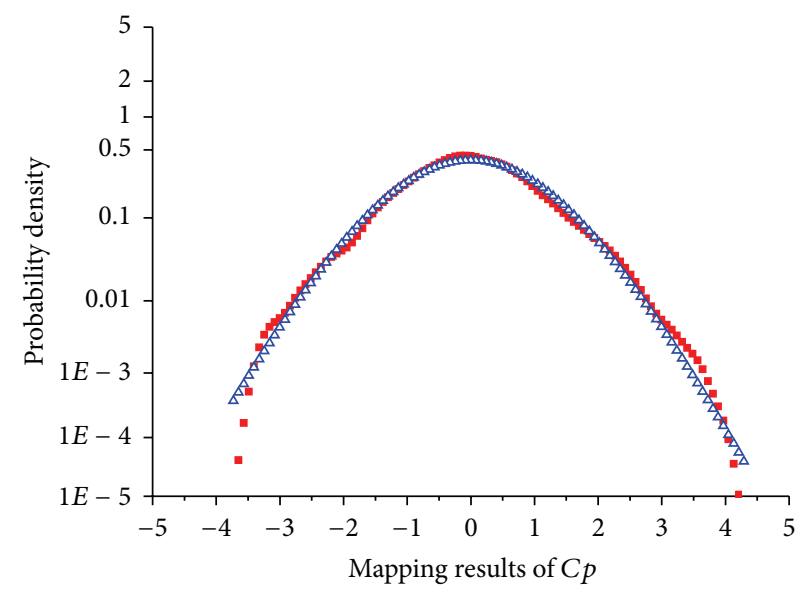

Wind pressure coefficients of tap U03 in wind angle 0 $\left(E_{C p}=-0.64 ; s_{C p}=0.23 ; C_{s-C p}=-1.51 ; C_{k-C p}=6.98\right)$

- Mapping results

$\triangle$ Standard gaussian process

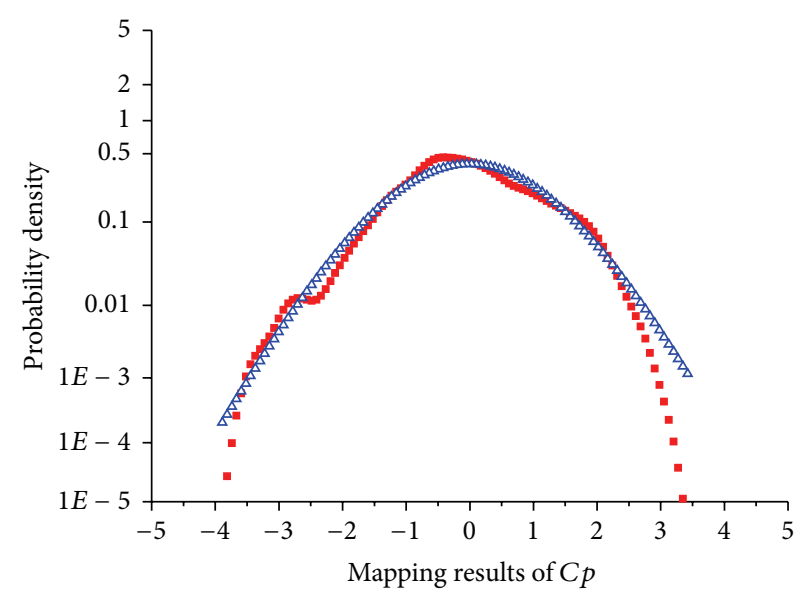

Wind pressure coefficients of tap U05 in wind angle 45 $\left(E_{C p}=-0.51 ; s_{C p}=0.17 ; C_{s-C p}=-2.42 ; C_{k-C p}=14.64\right)$

- Mapping results

$\Delta$ Standard gaussian process

FIGURE 7: The comparative results of representative test taps on surfaces of the high-rise building model.

According to statistical theory, the probability density formula of a one-dimensional random variable function $y=$ $g(x)$ is

$$
f_{Y}(y)=f_{X}\left(g^{-1}(y)\right) *\left|\left[g^{-1}(y)\right]^{\prime}\right|
$$

Using a reduced variable, $y=\left(x_{e}-\mu\right) / \sigma$, substituted into (5) and (7), the following expression is obtained:

$$
p_{Y}(y)=\exp \left\{-[1-k y]^{1 / k}\right\} *[1-k y]^{1 / k-1}
$$

Define another reduced variable

$$
z=[1-k y]^{1 / k} .
$$

And then substituting it into (8) and in (7), another expression is obtained:

$$
p_{Z}(z)=\exp (-z)
$$

From $z=[1-k y]^{1 / k}$,

$$
E[1-k y]=E\left[z^{k}\right]=\int_{0}^{\infty} e^{-z} z^{k} d z=\Gamma(k+1)
$$

Substituting the reduced variable $y=\left(x_{e}-\mu\right) / \sigma$ and rearranging the terms yield

$$
\overline{x_{e}}=\mu+\sigma * \frac{1-\Gamma(k+1)}{k} .
$$




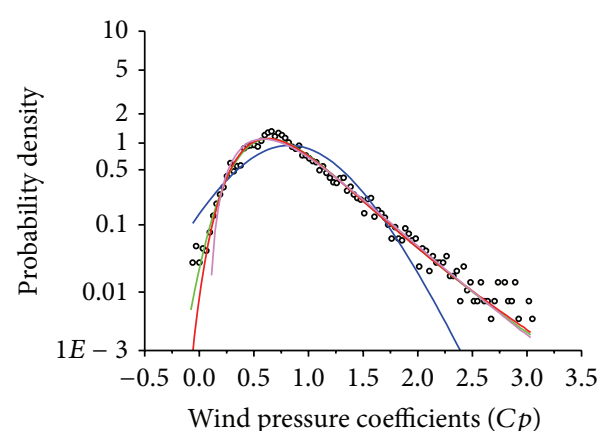

_ Gaussian distribution __ Lognormal distribution

- GEV distribution _ Gamma distribution

- Wind pressure coefficients of tap 113 in wind angle 0

$\left(E_{C p}=0.83 ; s_{C p}=0.42 ; C_{s-C p}=1.14 ; C_{k-C p}=5.14\right)$

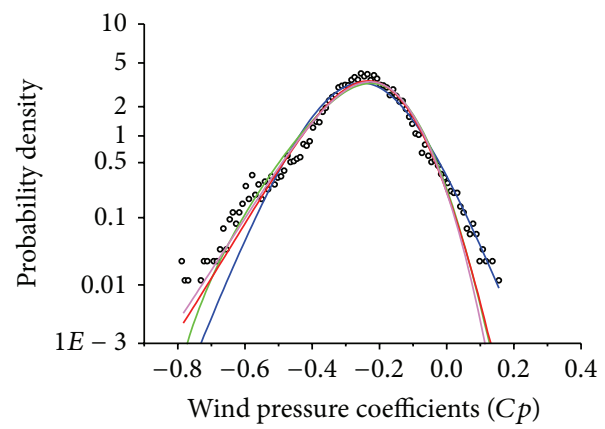

_ Gaussian distribution __ Lognormal distribution

- GEV distribution - Gamma distribution

- Wind pressure coefficients of tap 184 in wind angle 0

$\left(E_{C p}=-0.25 ; s_{C p}=0.12 ; C_{s-C p}=-0.43 ; C_{k-C p}=3.97\right)$

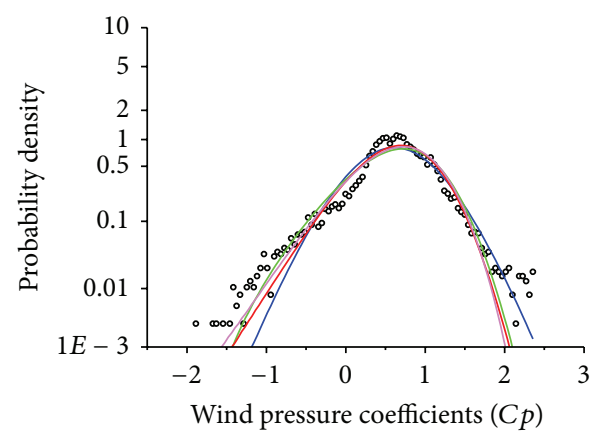

$\begin{array}{ll}\text { - Gaussian distribution } & \text { Lognormal distribution } \\ \text { GEV distribution } & \text { Gamma distribution }\end{array}$

- Wind pressure coefficients of tap 110 in wind angle 45

$\left(E_{C p}=0.61 ; s_{C p}=0.49 ; C_{s-C p}=0.46 ; C_{k-C p}=4.64\right)$

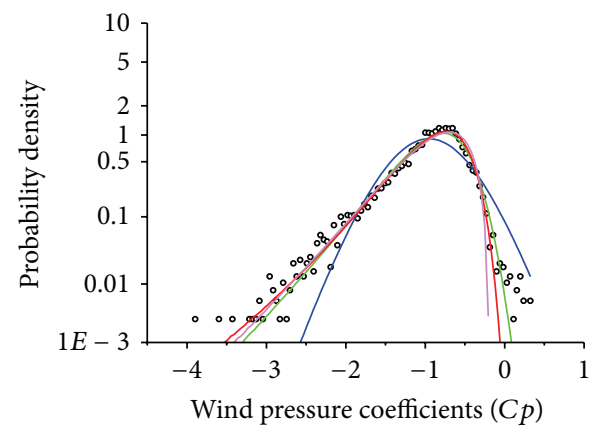

Gaussian distribution _ Lognormal distribution

GEV distribution - Gamma distribution

- Wind pressure coefficients of tap 138 in wind angle 0

$\left(E_{C p}=-0.95 ; s_{C p}=0.44 ; C_{s-C p}=-1.16 ; C_{k-C p}=5.33\right)$

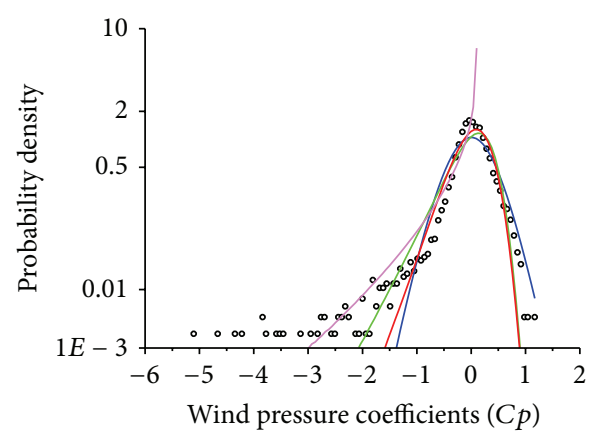

$\begin{array}{ll}\text { Gaussian distribution } & \text { Lognormal distribution } \\ \text { GEV distribution } & \text { Gamma distribution }\end{array}$

- Wind pressure coefficients of tap 85 in wind angle 45

$\left(E_{C p}=0.003 ; s_{C p}=0.37 ; C_{s-C p}=-2.86 ; C_{k-C p}=27.12\right)$

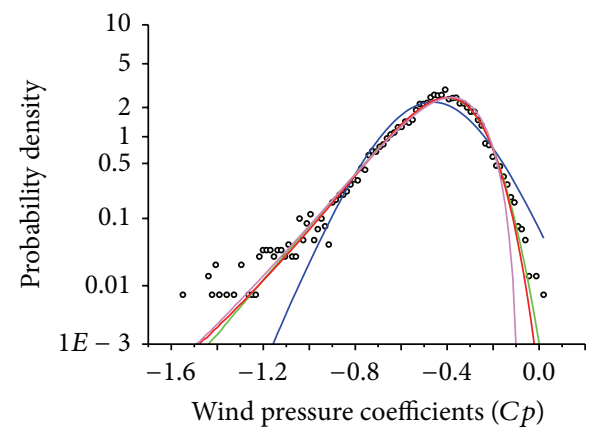

$\begin{array}{ll}\text { - Gaussian distribution } & \text { Lognormal distribution } \\ \text { GEV distribution } & \text { Gamma distribution }\end{array}$

- Wind pressure coefficients of tap 191 in wind angle 45

$\left(E_{C p}=-0.46 ; s_{C p}=0.18 ; C_{s-C p}=0.94 ; C_{k-C p}=5.02\right)$

FIGURE 8: Comparison of results of fitting with the probability distribution for test taps on the surfaces of low-rise building model at 0 and $45^{\circ}$ wind angle tests.

If $k$ is zero, then (12) is reduced to (13), which is the formula for the predicted extreme values based on the extreme value type I distribution:

$$
\overline{x_{e}}=\mu+\gamma * \sigma
$$

where $\gamma=0.5772$, the Euler's constant.
4.2. Relationship between the Parameters of the GEV Distribution and the Observation Length. Provided that a test data sample with an appropriate length of time, $t_{1}$, needs to be divided into several sub-samples with a length of time $t_{2}$, where $t_{1} \geq t_{2}$, then the number of sub-samples, $n=t_{1} / t_{2}$, and $n$ extreme values can be obtained. Consider that, within 


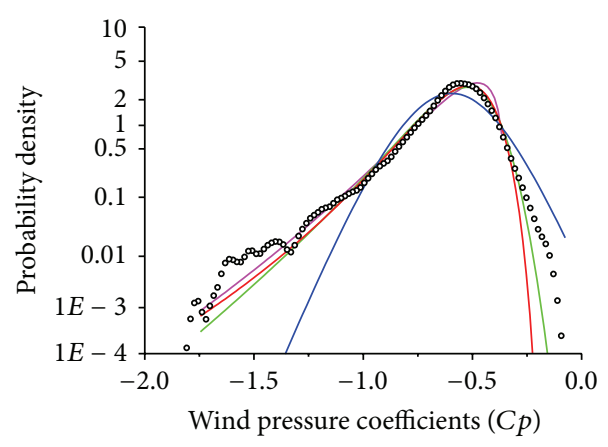

— Gamma distribution __ Lognormal distribution GEV distribution _ Gaussian distribution

- Wind pressure coefficients of tap M03 in wind angle 0

$\left(E_{C p}=-0.60 ; s_{C p}=0.17 ; C_{s-C p}=-1.40 ; C_{k-C p}=7.04\right)$

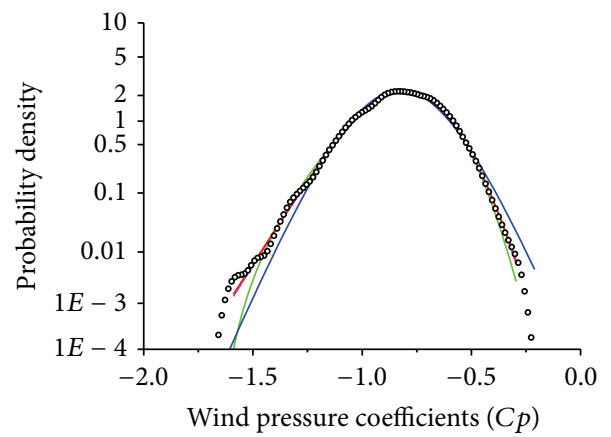

- Gamma distribution _ Lognormal distribution - GEV distribution _ Gaussian distribution

- Wind pressure coefficients of tap M13 in wind angle 0 $\left(E_{C p}=0.82 ; s_{C p}=0.17 ; C_{s-C p}=0.27 ; C_{k-C p}=3.03\right)$
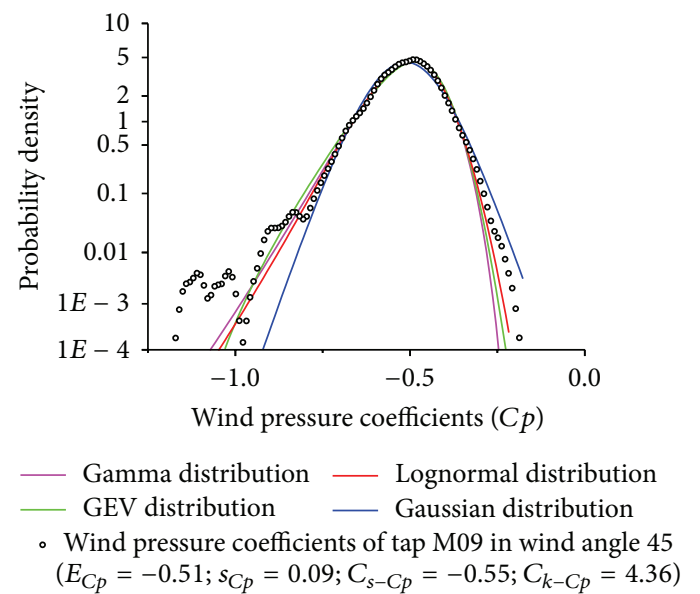

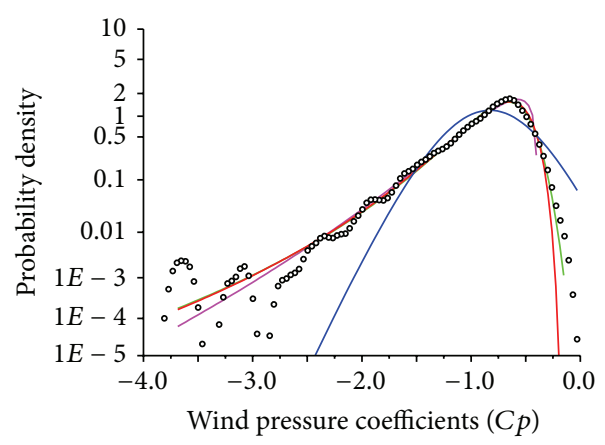

_ Gamma distribution _ Lognormal distribution GEV distribution _ Gaussian distribution

- Wind pressure coefficients of tap M09 in wind angle 0

$\left(E_{C p}=-0.83 ; s_{C p}=0.33 ; C_{s-C p}=-1.54 ; C_{k-C p}=7.82\right)$

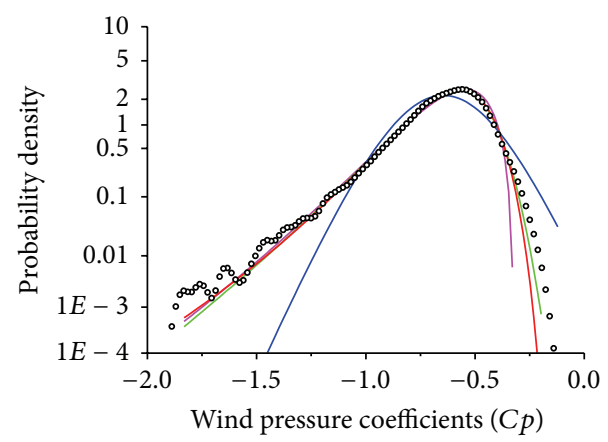

— Gamma distribution — Lognormal distribution GEV distribution _ Gaussian distribution

- Wind pressure coefficients of tap A29 in wind angle 45

$\left(E_{C p}=-0.65 ; s_{C p}=0.18 ; C_{s-C p}=-1.12 ; C_{k-C p}=5.56\right)$

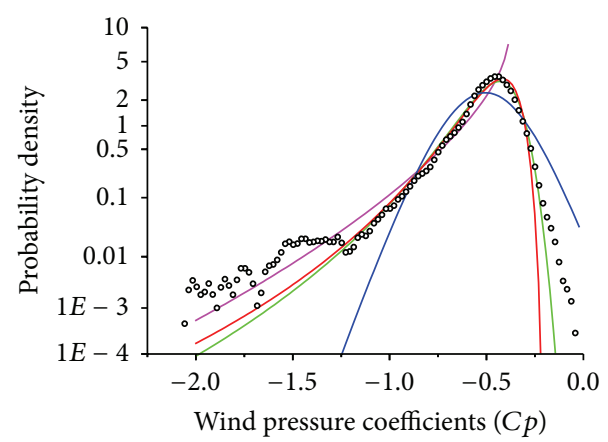

— Gamma distribution _ Lognormal distribution

- GEV distribution _ Gaussian distribution

- Wind pressure coefficients of tap U05 in wind angle 45

$\left(E_{C p}=-0.51 ; s_{C p}=0.17 ; C_{s-C p}=-2.42 ; C_{k-C p}=14.64\right)$

FIGURE 9: Comparison of results of fitting with the probability distribution for test taps on the surfaces of high-rise building model at 0 and $45^{\circ}$ wind angle tests.

$t_{1}$, the probability $F\left(x_{e 1}\right)$ is not less than $x$, whereas, within $t_{2}$, the probability $F\left(x_{e 2}\right)$ is not less than $x$. Supposing that the extreme values within $t_{2}$ are independent from each other, the following equation can be obtained:

$$
F\left(x_{e 1}\right)=F^{n}\left(x_{e 2}\right) .
$$

Given that all the observed extreme values of any random process fit the GEV distribution, substituting (4) in (14) yields

$$
\exp \left\{-\left[1-k_{1} y_{1}\right]^{1 / k_{1}}\right\}=\exp \left\{-n\left[1-k_{2} y_{2}\right]^{1 / k_{2}}\right\}
$$




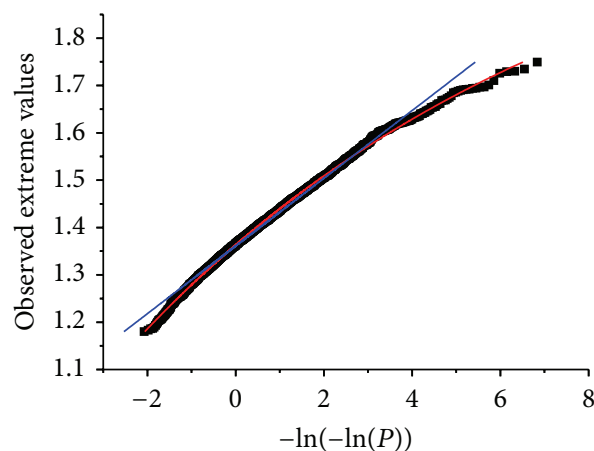

Positive extreme at tap $\mathrm{N} 13$ for $0^{\circ}$ wind angle

- Observed extreme values

- GEV distribution $(k=0.0977, s=0.0802, u=1.3631)$

- Gumbel distribution $(s=0.0715, u=1.3610)$

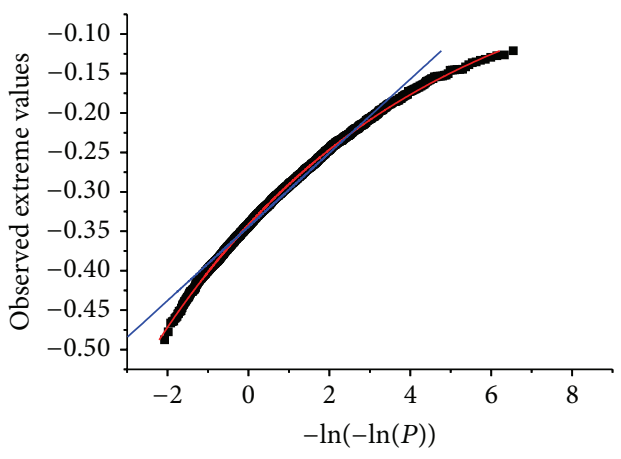

Negative extreme at tap $\mathrm{H} 09$ for $0^{\circ}$ wind angle

- Observed extreme values

- GEV distribution $(k=0.1556, s=0.0554, u=-0.3417)$

- Gumbel distribution $(s=0.0468, u=-0.3442)$

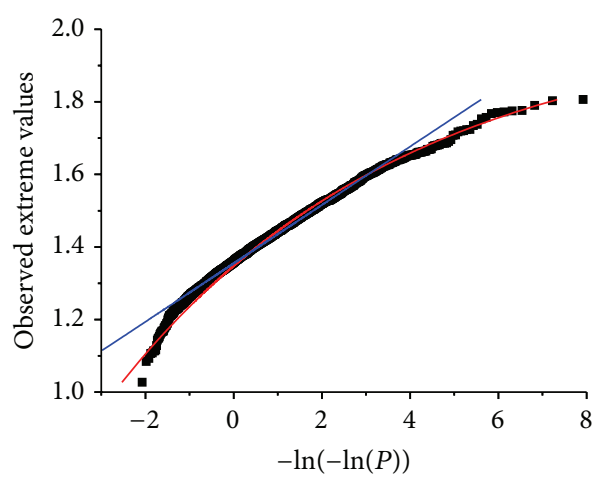

Positive extreme at tap I15 for $45^{\circ}$ wind angle

- Observed extreme values

- GEV distribution $(k=0.1524, s=0.1039, u=1.3476)$

— Gumbel distribution $(s=0.0805, u=1.3547)$

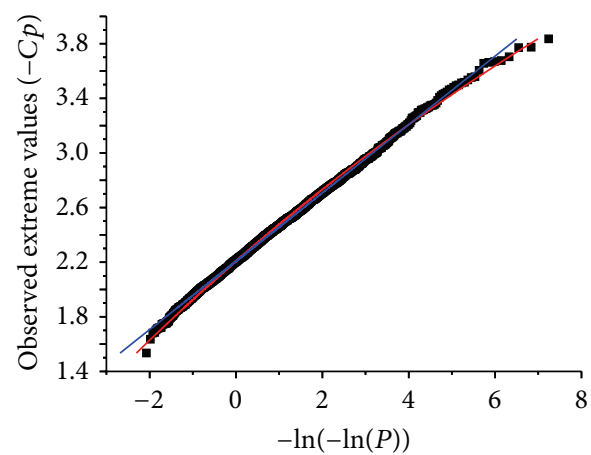

Negative extreme at tap N09 for $0^{\circ}$ wind angle

- Observed extreme values

- GEV distribution $(k=0.0495, s=0.2755, u=2.2071)$

— Gumbel distribution $(s=0.2505, u=2.2064)$

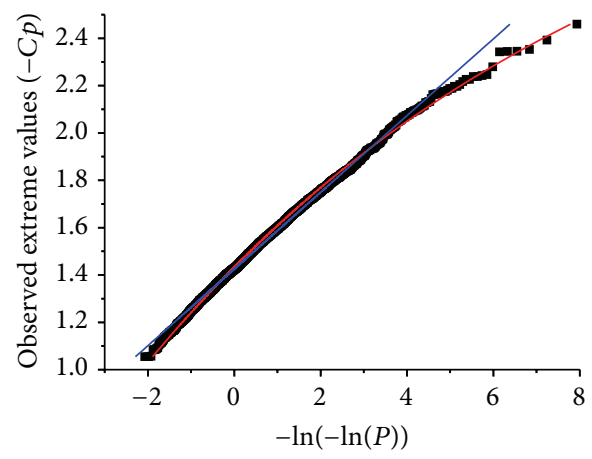

Negative extreme at tap $\mathrm{O} 03$ for $0^{\circ}$ wind angle - Observed extreme values

- GEV distribution $(k=0.0902, s=0.1835, u=1.4331)$

- Gumbel distribution $(s=0.1621, u=1.4252)$

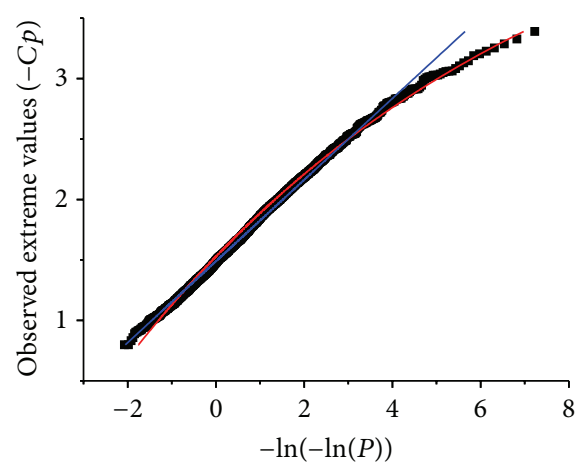

Negative extreme at tap U06 for $45^{\circ}$ wind angle

- Observed extreme values

- GEV distribution $(k=0.1065, s=0.3781, u=1.5279)$

_ Gumbel distribution $(s=0.3366, u=1.4890)$

FIGURE 10: Comparison of fitting results of large number of samples for test taps on the surfaces of high-rise building model. 


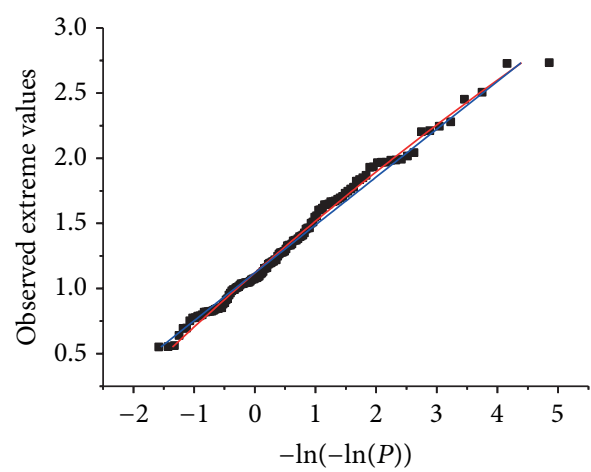

Positive extreme at tap 113 for $0^{\circ}$ wind angle

- Observed extreme values

- GEV distribution $(k=-0.0472, s=0.406, u=1.1201)$

- Gumbel distribution $(s=0.3674, u=1.1202)$

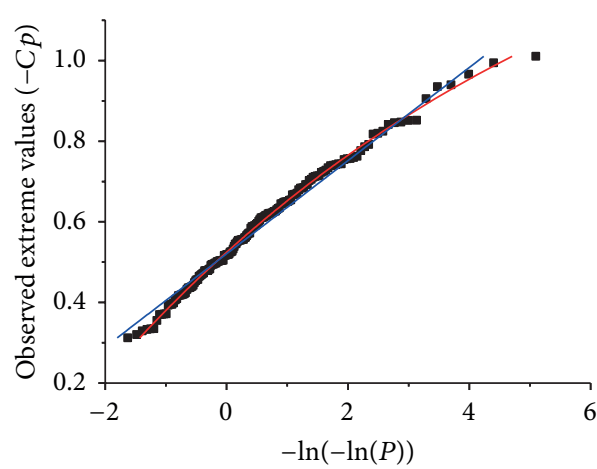

Positive extreme at tap 189 for $0^{\circ}$ wind angle

- Observed extreme values

- GEV distribution $(k=0.1215, s=0.1356, u=0.5243)$

- Gumbel distribution $(s=0.1155, u=0.5206)$

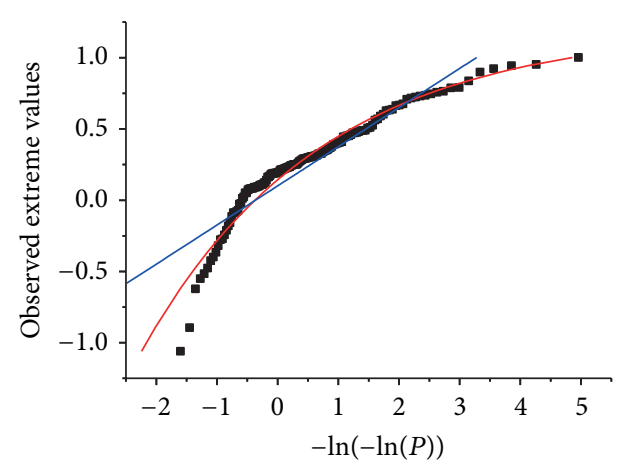

Positive extreme at tap 73 for $45^{\circ}$ wind angle

- Observed extreme values

GEV distribution $(k=-0.3380, s=0.3598, u=0.1428)$

Gumbel distribution $(s=0.2748, u=0.1005)$

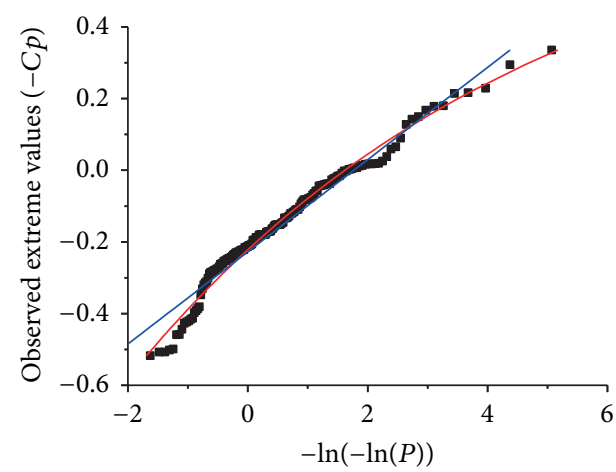

Negative extreme at tap 113 for $0^{\circ}$ wind angle - Observed extreme values

- GEV distribution $(k=0.1475, s=0.1541, u=-0.2220)$

- Gumbel distribution $(s=0.1287, u=-0.2275)$

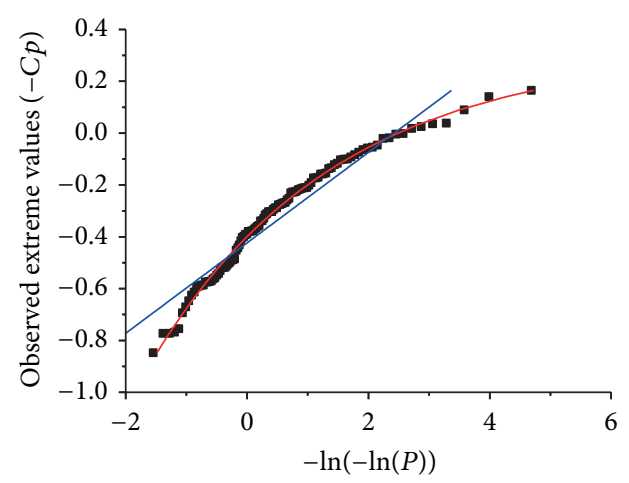

Negative extreme at tap 146 for $0^{\circ}$ wind angle - Observed extreme values

- GEV distribution $(k=0.3246, s=0.2344, u=-0.3994)$

- Gumbel distribution

$$
(s=0.1745, u=-0.4232)
$$

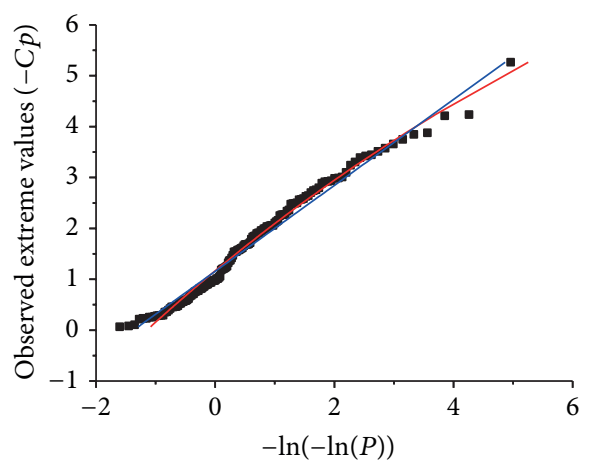

Negative extreme at tap 73 for $45^{\circ}$ wind angle

- Observed extreme values

- GEV distribution $(k=0.0853, s=0.9680, u=1.1669)$

- Gumbel distribution $(s=0.8440, u=1.1565)$

FIGURE 11: Comparison of fitting results of single sample for test taps on the surfaces of low-rise building model. 


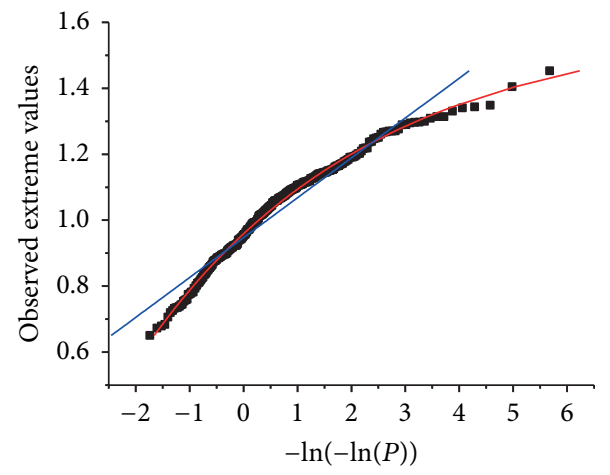

Positive extreme at tap N13 for $0^{\circ}$ wind angle

- Observed extreme values

- GEV distribution

$(k=0.2371, s=0.1518, u=0.9589)$

- Gumbel distribution $(s=0.1209, u=0.9472)$

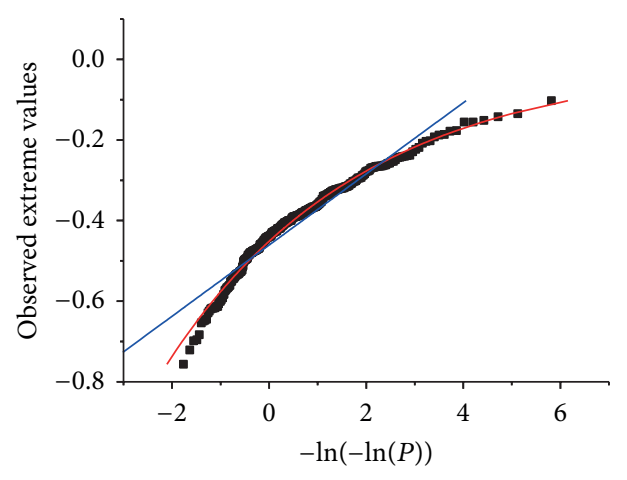

Positive extreme at tap U03 for $0^{\circ}$ wind angle

- Observed extreme values

- GEV distribution $(k=0.2474, s=0.1104, u=-0.4513)$

— Gumbel distribution $(s=0.0883, u=-0.4608)$

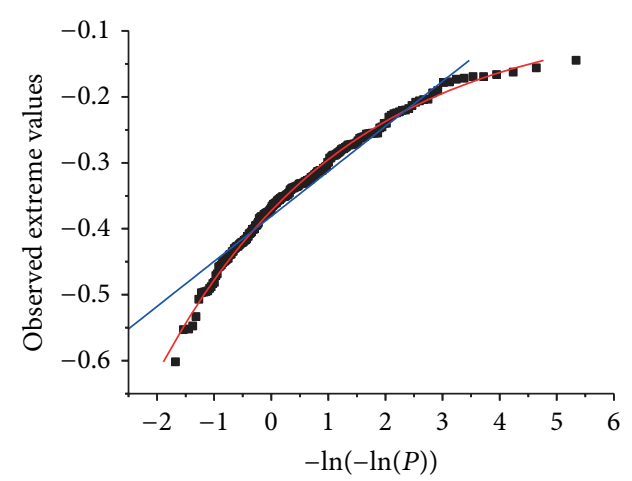

Positive extreme at tap U06 for $45^{\circ}$ wind angle

- Observed extreme values

GEV distribution

$(k=0.3006, s=0.0901, u=-0.3728)$

— Gumbel distribution

$(s=0.0682, u=-0.3812)$

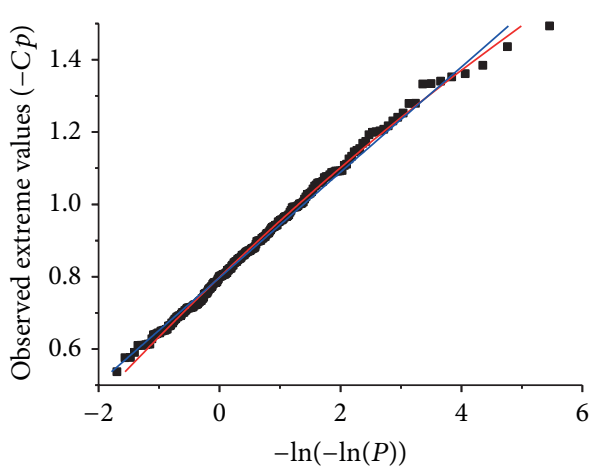

Negative extreme at tap $\mathrm{I} 03$ for $0^{\circ}$ wind angle

- Observed extreme values

- GEV distribution $(k=0.0578, s=0.1602, u=0.7986)$

- Gumbel distribution $(s=0.1457, u=0.7974)$

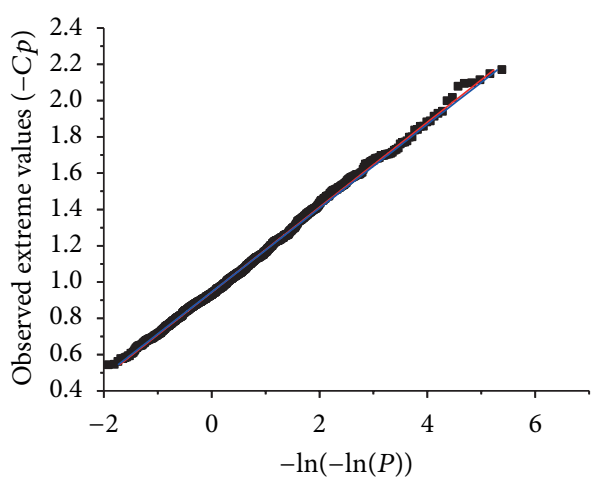

Negative extreme at tap $\mathrm{H} 09$ for $0^{\circ}$ wind angle

- Observed extreme values

- GEV distribution $(k=1.8392 * e-5, s=0.2342, u=0.9437)$

- Gumbel distribution $(s=0.2312, u=0.9448)$

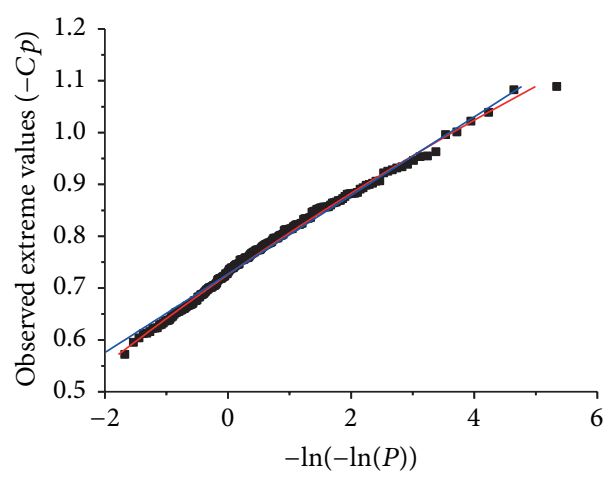

Negative extreme at tap $\mathrm{H} 03$ for $45^{\circ}$ wind angle

- Observed extreme values

- GEV distribution $(k=0.0528, s=0.0826, u=0.7260)$

— Gumbel distribution $(s=0.0758, u=0.7271)$

FIGURE 12: Comparison of fitting results of single sample for test taps on the surfaces of high-rise building model. 


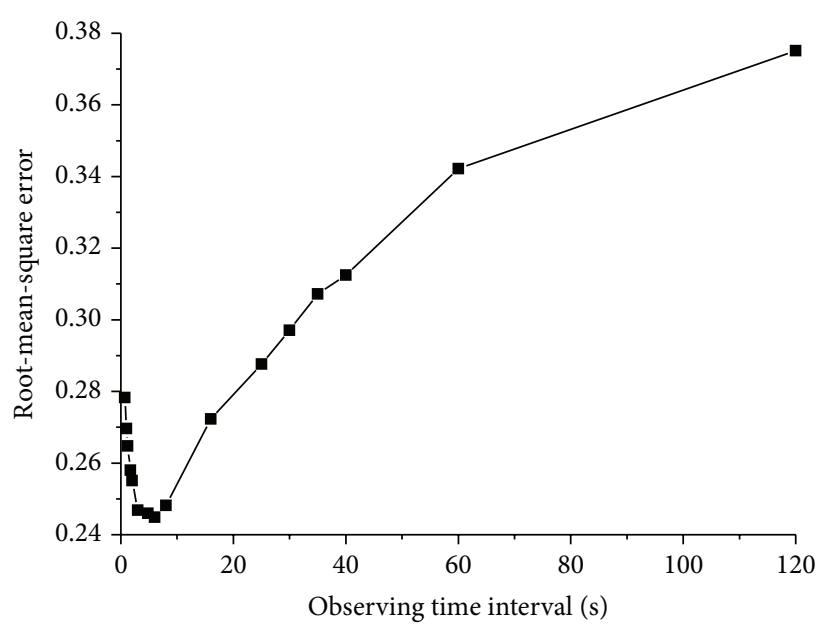

(a) Root-mean-square error

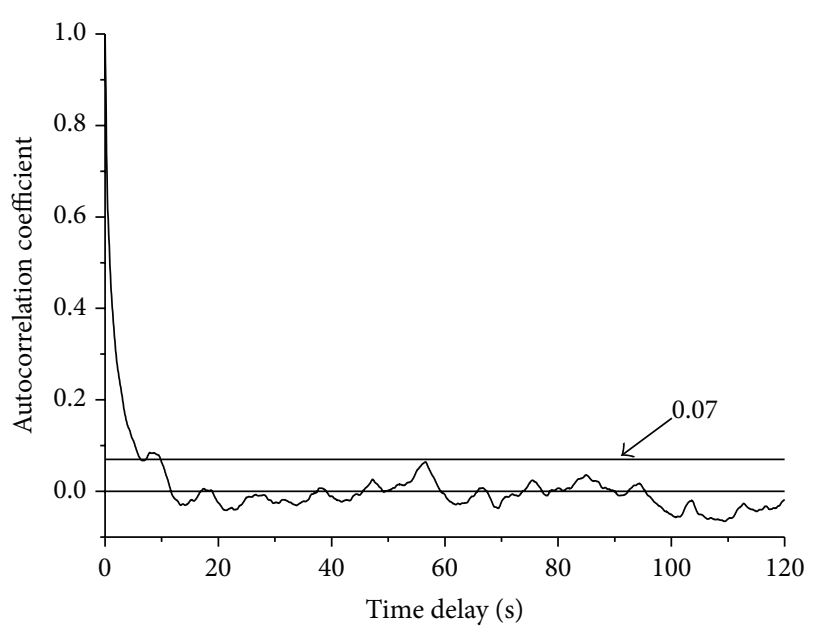

(b) Autocorrelation coefficients

FIGURE 13: Relationship between the root-mean-square error of the estimated extreme values and the observation period with the mean value of the correlation coefficients of the wind pressure history.

where the subscript 1 corresponds to the observation length $t_{1}$ and the subscript 2 corresponds to the observation length $t_{2}$.

After rearranging, the following expression is obtained:

$$
\frac{\left(1-k_{1} y_{1}\right)^{k_{2} / k_{1}}}{1-k_{2} y_{2}}=n^{k_{2}}
$$

The numerator of the left part of (16) can be expressed as a Taylor expansion, supposing that $-1<k_{1} y_{1}<1$,

$$
\begin{aligned}
\left(1-k_{1} y_{1}\right)^{k_{2} / k_{1}}= & 1-k_{2} y_{1}+\frac{k_{2}\left(k_{2}-k_{1}\right)}{2 !}+\cdots \\
& +(-1)^{n} \frac{k_{2}\left(k_{2}-k_{1}\right) \cdots\left[k_{2}-(n-1) k_{1}\right]}{n !} y_{1}^{n} \\
& +\cdots .
\end{aligned}
$$

Substituting the reduced variable $y=\left(x_{e}-\mu\right) / \sigma$ and (17) into (16), and considering the dependence of the parameters $\mu, \sigma$, and the observation length, the following expression is obtained:

$$
C_{0}+C_{1} x_{e}+C_{2} x_{e}^{2}+C_{2} x_{e}^{3}+\cdots=0,
$$

where

$$
\begin{aligned}
C_{0}= & -n^{k_{2}}+1-\frac{n^{k_{2}} k_{2}}{\sigma_{2}} \mu_{2}+\frac{k_{2}}{\sigma_{1}} \mu_{1}+\frac{k_{2}\left(k_{2}-k_{1}\right)}{2 !} \frac{\mu_{1}^{2}}{\sigma_{1}^{2}} \\
& +\cdots+\frac{k_{2}\left(k_{2}-k_{1}\right) \cdots\left[k_{2}-(n-1) k_{1}\right]}{n !} \frac{\mu_{1}^{n}}{\sigma_{1}^{n}}+\cdots, \\
C_{1}= & \frac{n^{k_{2}} k_{2}}{\sigma_{2}}-\frac{k_{2}}{\sigma_{1}}-\frac{k_{2}\left(k_{2}-k_{1}\right)}{2 !} \frac{2 \mu_{1}}{\sigma_{1}^{2}}
\end{aligned}
$$

$$
\begin{aligned}
& -\cdots-\frac{k_{2}\left(k_{2}-k_{1}\right) \cdots\left[k_{2}-(n-1) k_{1}\right]}{n !} \frac{n \mu_{1}^{n-1}}{\sigma_{1}^{n}}-\cdots, \\
C_{2}= & \sum_{n=2}^{\infty} \frac{k_{2}\left(k_{2}-k_{1}\right) \cdots\left[k_{2}-(n-1) k_{1}\right]}{n ! \sigma_{1}^{n}} * C_{n}^{2} * \mu_{1}^{n-2} \\
= & \frac{k_{2}\left(k_{2}-k_{1}\right)}{2 \sigma_{1}^{2}}\left(1+\frac{k_{1} \mu_{1}}{\sigma_{1}}\right)^{\left(k_{2} / k_{1}\right)-2}, \\
C_{3}= & \sum_{n=3}^{\infty} \frac{k_{2}\left(k_{2}-k_{1}\right) \cdots\left[k_{2}-(n-1) k_{1}\right]}{n ! \sigma_{1}^{n}} * C_{n}^{3} * \mu_{1}^{n-3},
\end{aligned}
$$

Equation (18) is established permanently only if all the coefficients are simultaneously equal to zero; that is, the equations $C_{0}=0 ; C_{1}=0 ; C_{2}=0 ; C_{3}=0 ; \ldots$ are satisfied at the same time. Hence, the following solution satisfies these conditions

$$
\begin{gathered}
k_{1}=k_{2}=k, \\
\sigma_{1}=\frac{1}{n^{k}} \sigma_{2}, \\
\mu_{1}=\mu_{2}-\frac{1-n^{k}}{k n^{k}} \sigma_{2} .
\end{gathered}
$$

If the parameter $k$ is zero, then (21) and (22) are reduced to the parameter transform formulations based on the extreme values type I distribution,

$$
\begin{gathered}
\sigma_{1}=\sigma_{2}, \\
\mu_{1}=\mu_{2}+\ln (n) \sigma_{2} .
\end{gathered}
$$




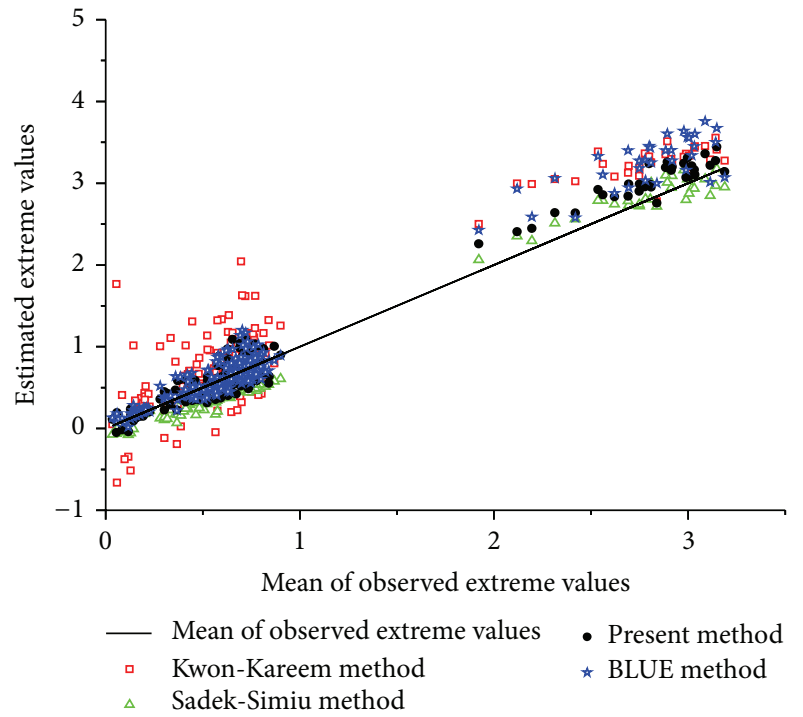

(a) $0^{\circ}$ wind angle

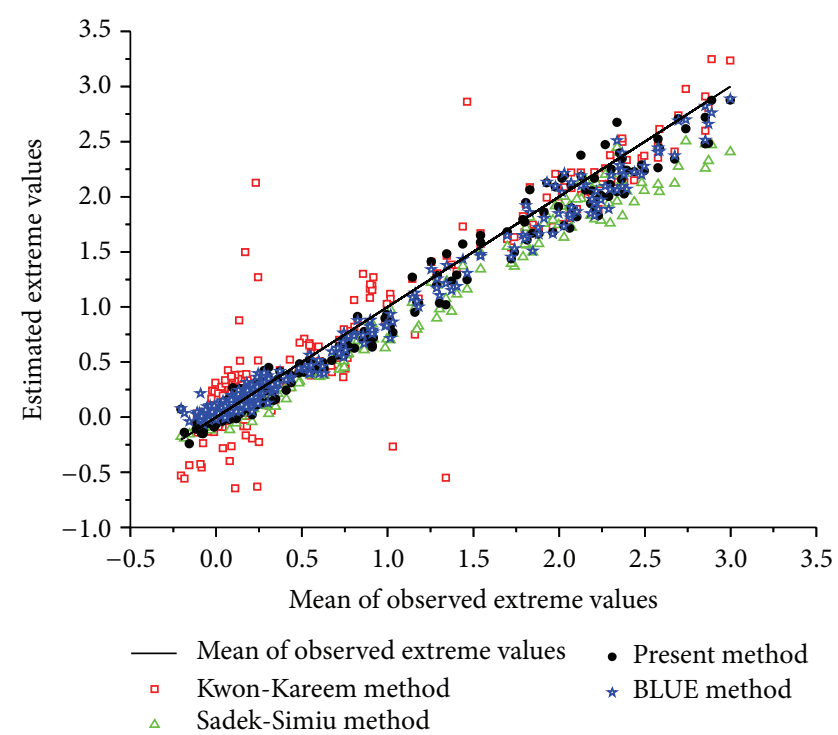

(b) $45^{\circ}$ wind angle

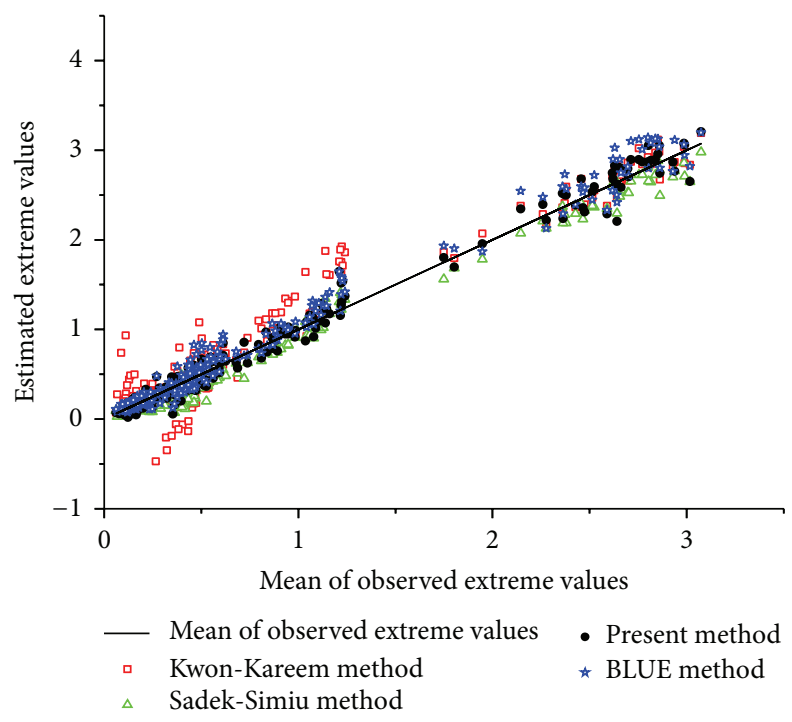

(c) $90^{\circ}$ wind angle

FIGURE 14: Comparison of estimated positive extreme values on low-rise building.

According to the above conversion, a sample with a duration of $t_{1}$ can be divided into $n\left(n=t_{1} / t_{2}\right)$ nonoverlapping sub-samples with a time length of $t_{2}$. From the probability distribution parameters of extreme values provided by the sub-samples, the extreme value distribution parameters of the sample can be obtained. The extreme values can be calculated using (12) or (13). Thus, estimation of the extreme values in a single sample with a time length $t_{1}$ can be done.

4.3. Optimal Observation Period of Subsamples. In the present estimating method, larger values of $n$ lead to more peak values used for estimating the extreme values. A more detailed survey of the time history data is considered such that estimates of the extreme values can represent the internal characteristic of the primitive history data. However, the length of the sub-sample observation is shorter if the number of the sub-samples increases. When the observations on the sub-samples are short enough, the extreme values from the neighboring sub-samples are dependent on each other, which is contrary to (14). To ensure the independence of the extreme values of the sub-samples and simultaneously to obtain as many sub-samples as possible, an autocorrelation analysis of the parent sample is performed and the shortest time delay for the auto-correlation coefficient decaying from 1 to 0.07 , close to zero, is taken as the optimal observation period for the sub-sample. As an example, the autocorrelation results for the test case data obtained at $0^{\circ}$ wind angle are presented in Figure 13. The relation between the root-mean-square error of the estimated extreme values and the observation 


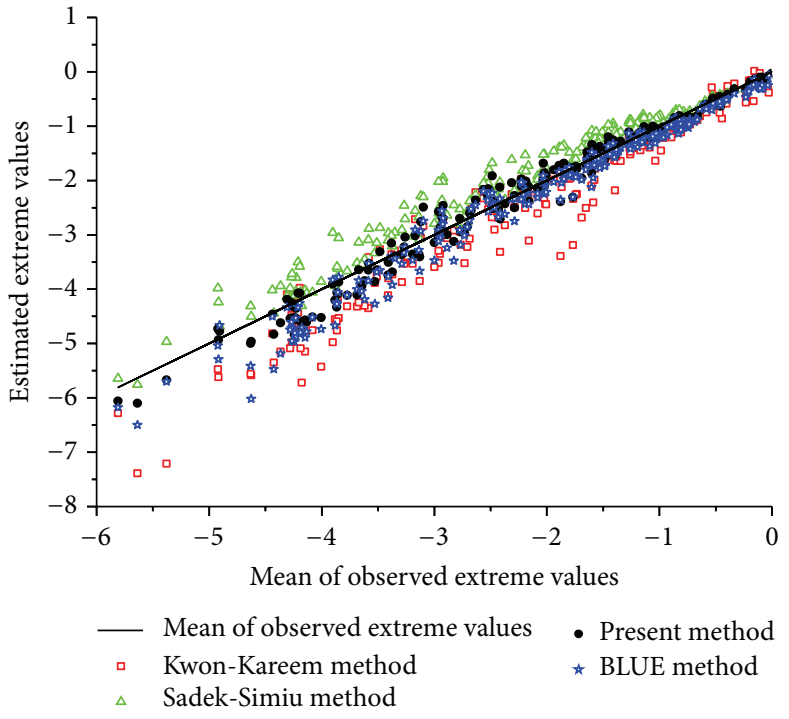

(a) $0^{\circ}$ wind angle

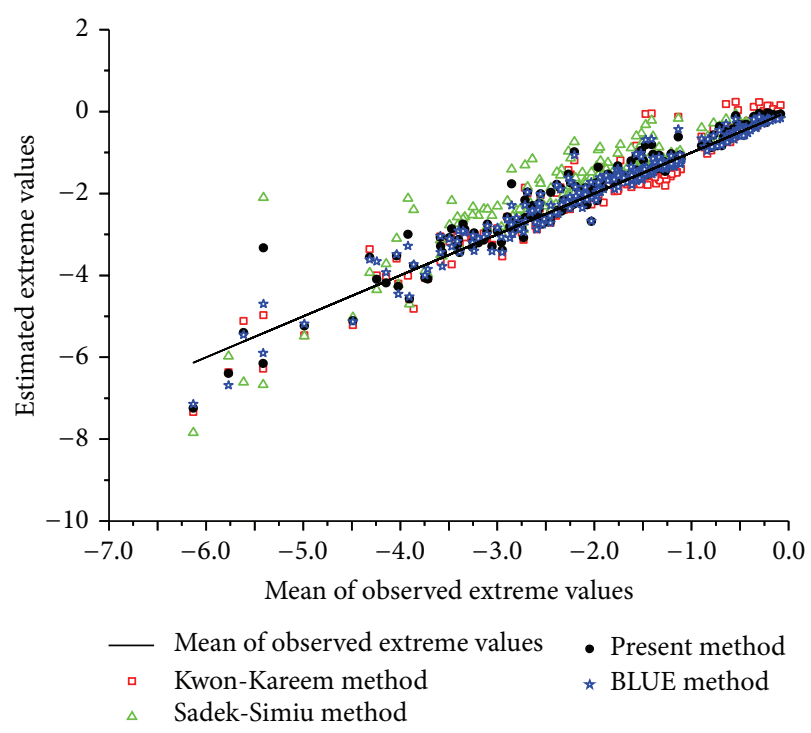

(b) $45^{\circ}$ wind angle

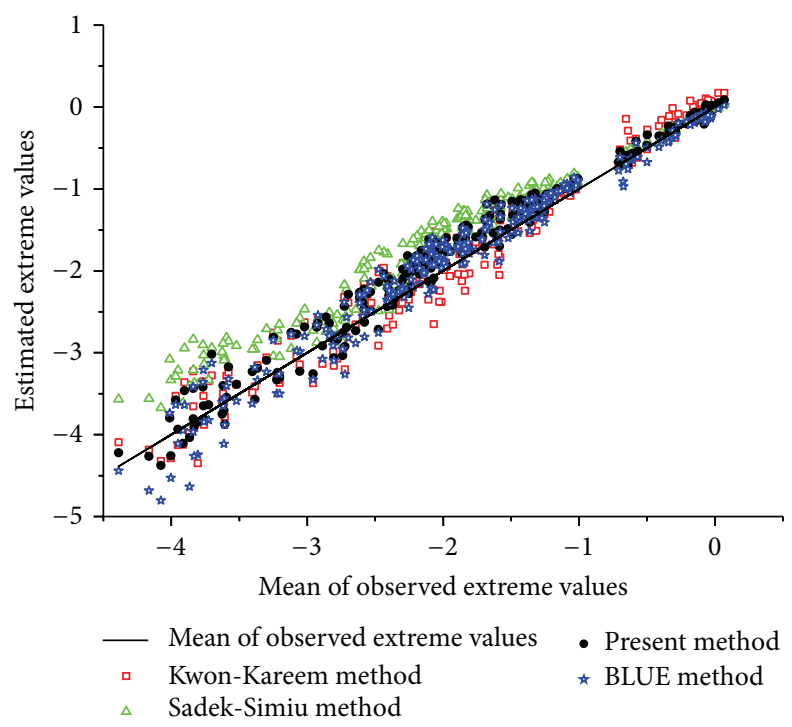

(c) $90^{\circ}$ wind angle

FIGURE 15: Comparison of estimated negative extreme values on low-rise building.

period of the wind pressure history is also shown in the figure. When the observation period for the samples is very short, the error of the estimated results is significant because the observed extreme values are not independent. With an increase in the observation period, the correlation among the observed extreme values of the neighboring subsamples weakens, and thereby reduces the error. When the observation period reaches about $6 \mathrm{~s}$, the error of the present method is minimal. However, with further increase in the observation period, the error increases because the decreased number of subsamples leads to an incomplete reflection of the intrinsic characteristics of the time history. Similarly, analysis of the auto-correlation of the time history shows that the shortest time delay for the auto-correlation coefficient to decay from 1 to 0.07 is also approximately $6 \mathrm{~s}$. More wind tunnel test data proved that the time period obtained by autocorrelation analysis is approximately the optimal observation period.

4.4. Calculating Procedure of the Present Method. To facilitate the comprehension and application of the present method, the procedure is listed below.

(1) The auto-correlation coefficients of the sample with a standard observation period $t_{1}$ (such as $10 \mathrm{~min}$ or $1 \mathrm{~h}$ ) were analyzed, and the shortest time delay $t_{2}$ for the auto-correlation coefficient to decay from 1 to 0.07 was obtained.

(2) The parent sample was divided into $n\left(n=t_{1} / t_{2}\right)$ subsamples with an observation period of $t_{2}$. 


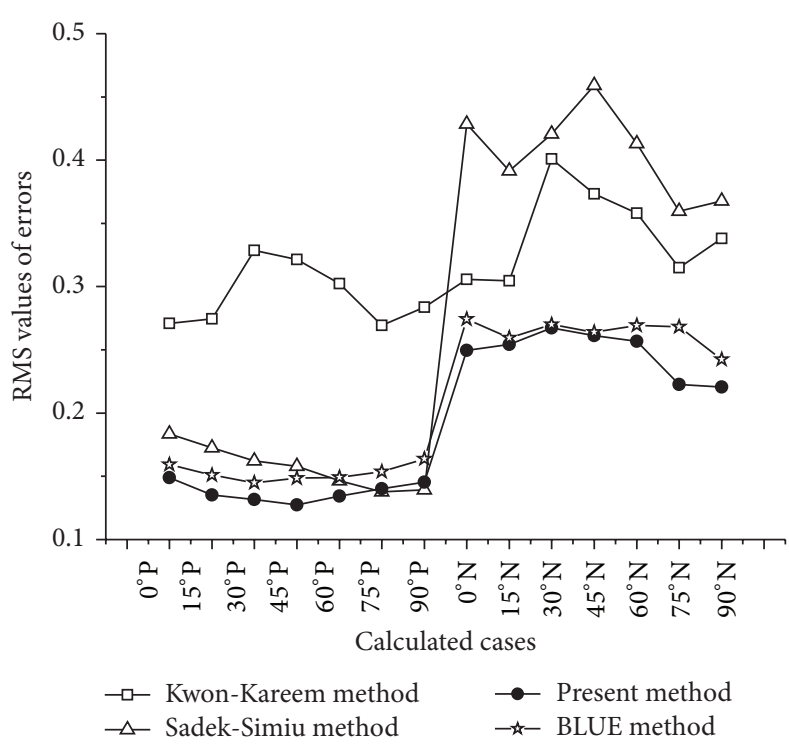

(a) Root-mean-square values of errors

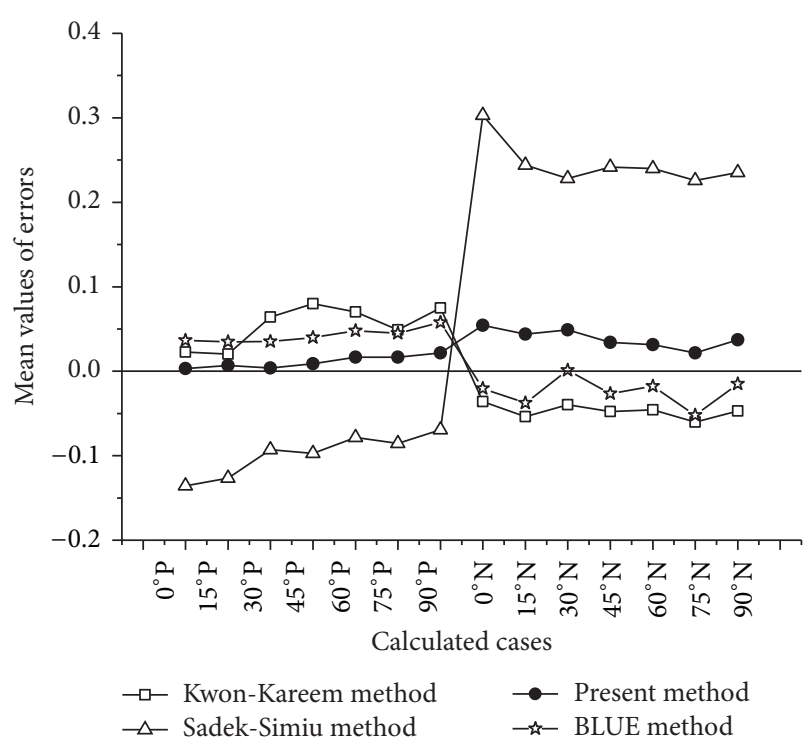

(b) Mean values of errors

FIGURE 16: Accuracy of the different methods under various working conditions of lowing (note that P represents the positive extreme values of calculated cases and $\mathrm{N}$ represents the negative extreme values of calculated cases).

(3) The maximum values of $n$ sub-samples were extracted and fitted with the GEV distribution using (4), and the extreme distribution parameters $k_{2}, \mu_{2}$, and $\sigma_{2}$ were obtained. If the fitting result of $k$ is less than zero, then these extreme values must be fitted again with the extreme value type I distribution in (5) to obtain the corresponding extreme distribution parameters $\mu_{2}$ and $\sigma_{2}$.

(4) The parameters for the observation period of $t_{2}\left(k_{2}\right.$, $\mu_{2}$, and $\left.\sigma_{2}\right)$ were transformed into those for $t_{1}\left(k_{1}\right.$, $\mu_{1}$, and $\sigma_{1}$ ) by (20), (21), and (22). Considering the condition for the extreme value type I distribution, the limit forms (23) were needed to transform the parameters for $t_{2}$ to those for $t_{1}$.

(5) The expected extreme values of $t_{1}$ were calculated using either (12) or (13).

For the calculation of negative values, the sign of the extreme values could be changed to transform the problem into a calculation of positive values; afterward, the problem could be solved by following the aforementioned procedure.

\section{Comparison between the Present Method and Previous Methods}

The BLUE, Kwon-Kareem, and Sadek-Simiu methods are presently the most commonly used methods to estimate extreme values. These methods, as well as the present method, were applied to estimate the extreme values of wind pressure in a standard sample. The accuracy of the different methods was determined by comparing the aforementioned methods. However, before the step of comparison of the four methods, the first problem is to determine the standard values. For the case of low-rise building, the mean value of 15 independent observed extreme values of 15 samples was considered as the standard extreme value while the mean value of 2800 independent observed extreme values of the 2800 samples was taken as the standard extreme value for the highrise building case. According to the instructions above, the MATLAB program is written. During the estimation by the Sadek-Simiu method, the level-crossing rate of the translated standard Gaussian time period $y(t)$ was approximately equal to that of the raw time period $x(t)$. Moreover, Grigoriu [20] revealed that the error from this approximation is negligible. The conclusion by Sadek and Simiu [19] that the distribution of the parent samples fits the gamma distribution with three parameters and Gaussian distribution is cited here. During the estimation by the Kwon-Kareem method, an improvement in the evaluation of a non-Gaussian process by the Hermite model of polynomial coefficients ( $h 3$ and $h 4$ ) was adopted as well as a modified calculating expression about the peak factor.

In this part, the estimating results of the four methods will be illustrated. In order to make it more convenient to explain the match condition between the estimating results $(E)$, the standard extreme values $(S)$, the mean value $(M)$, and the root-mean-square value (RMS) of errors were defined separately as follows:

$$
\begin{aligned}
M & =\frac{1}{n} \sum_{i=1}^{n}\left(\frac{1}{m} \sum_{j=1}^{m}(E-S)\right), \\
\mathrm{RMS} & =\sqrt{\frac{1}{n} \sum_{i=1}^{n}\left(\frac{1}{m} \sum_{j=1}^{m}(E-S)^{2}\right)},
\end{aligned}
$$




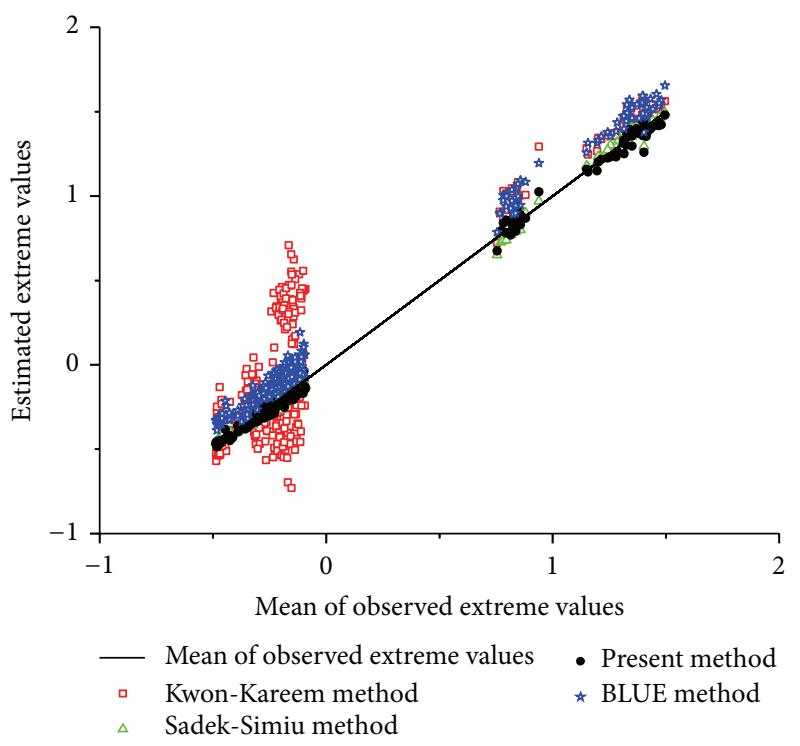

(a) $0^{\circ}$ wind angle

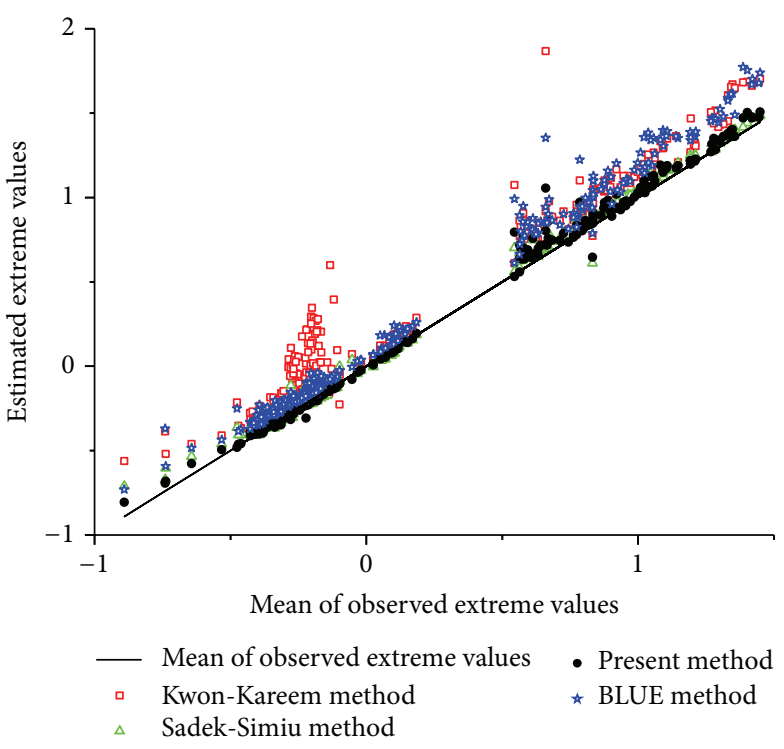

(b) $45^{\circ}$ wind angle

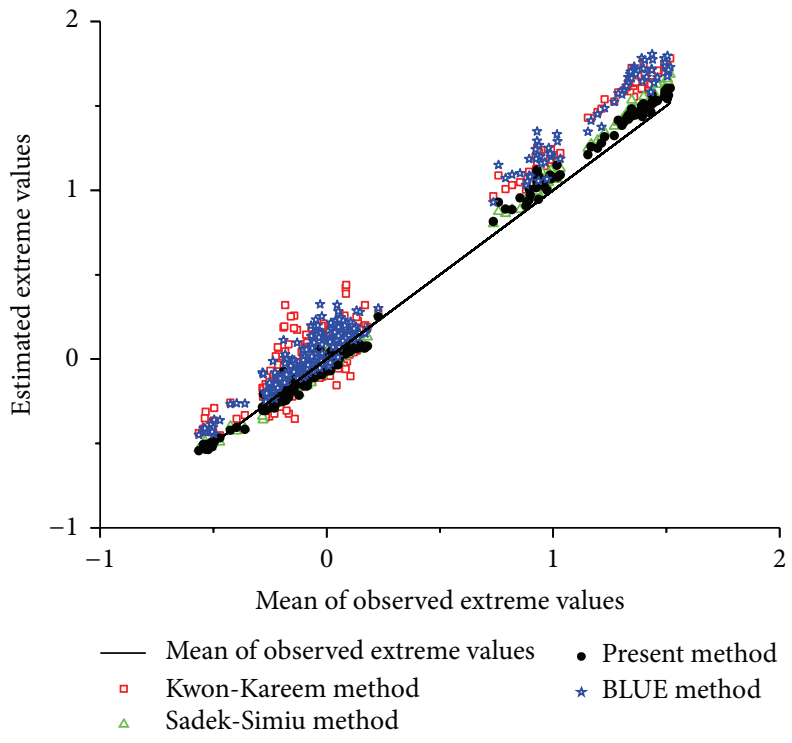

(c) $90^{\circ}$ wind angle

FIGURE 17: Comparison of estimated positive extreme values on high-rise building.

where $n=15$ is the number of samples of each tap and $m$ is the number of taps arranged on the surfaces of building models, 428 for the low-rise building model and 445 for the high-rise building model.

Results obtained for the test cases of 0,45 , and $90^{\circ}$ wind angles and the estimated negative wind pressure and positive wind pressure of the low-rise building are presented in Figures 14 and 15, respectively. The results obtained from the Kwon-Kareem method severely deviate from the average observed extreme values. The SadekSimiu method is inclined to be dangerous while the results of the BLUE method and the present method showed a good performance in Figure 14. Considering the negative wind pressure in Figure 15, the divergence of the results of the Kwon-Kareem method is still very large while the results obtained from the Sadek-Simiu method have large errors from the average observed extreme values. Fortunately, the results of the present method are very close to the average observed extreme values in these test cases; the data are evenly distributed about the sides of the average observed extreme values and have small divergence.

Figure 16 shows the root-mean-square values (Figure 16(a)) and mean values (Figure 16(b)) of errors of the five methods under 14 test cases. The computed results using additional test data indicate that the present method can have a fair estimate of the positive and negative extreme values of the wind pressure. 


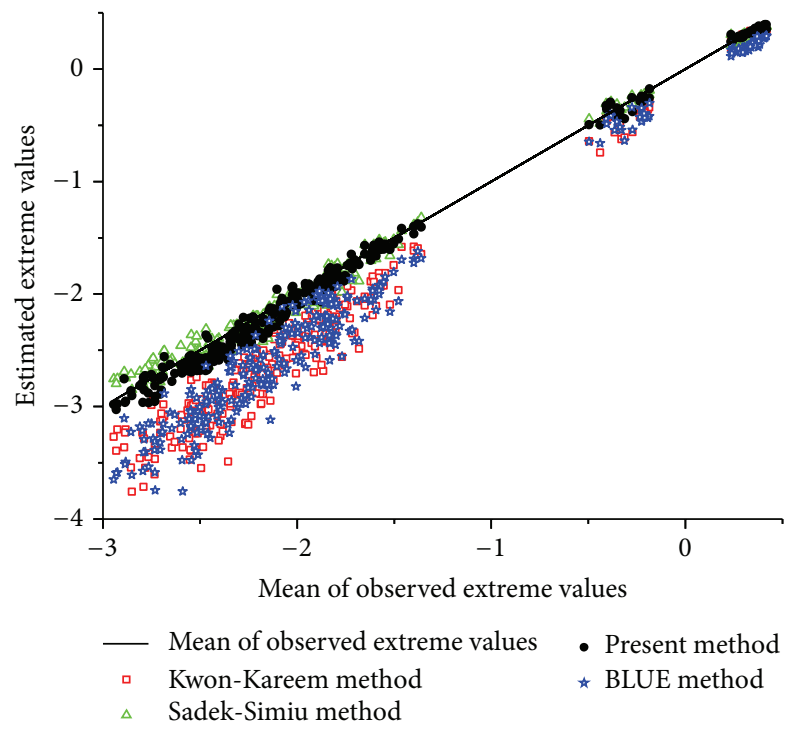

(a) $0^{\circ}$ wind angle

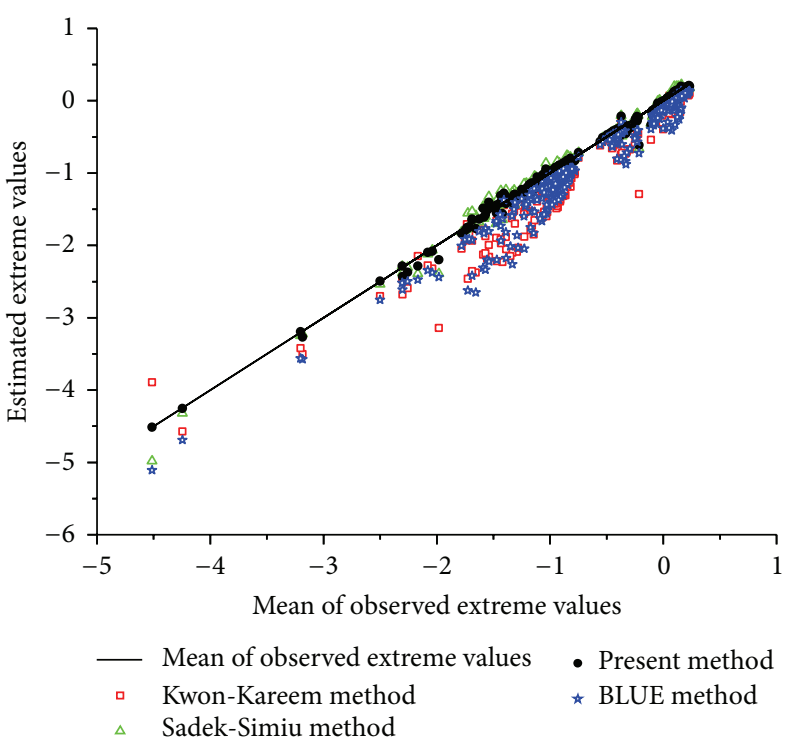

(b) $45^{\circ}$ wind angle

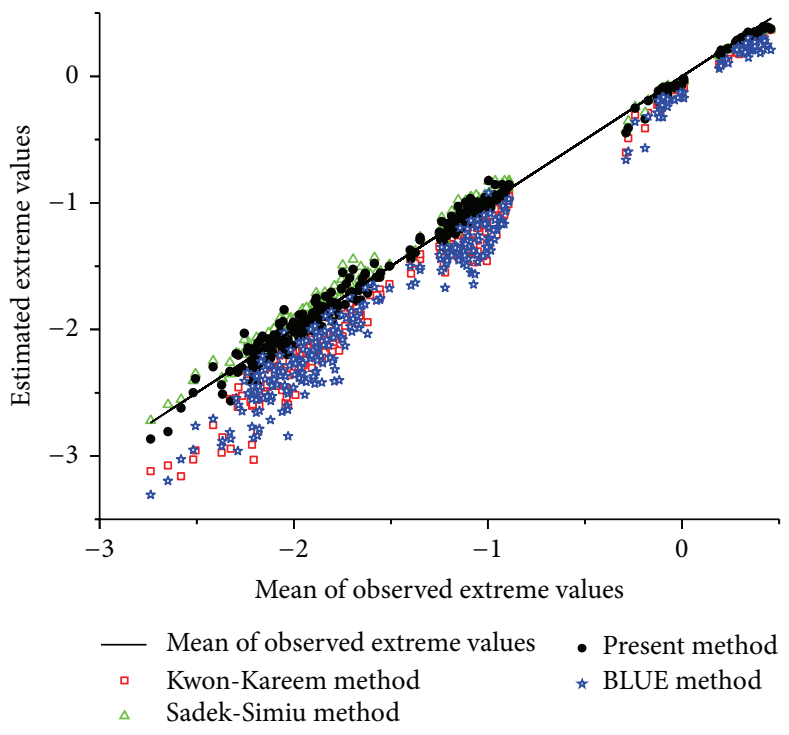

(c) $90^{\circ}$ wind angle

FIGURE 18: Comparation of estimated negative extreme values on high-rise building.

Results obtained for the test cases of 0,45 , and $90^{\circ}$ wind angles and the estimated positive wind pressure and negative wind pressure of the high-rise building are presented in Figures 17 and 18, respectively. It can be seen from the figure that the results estimated by present method distribute around the standard values evenly, with a small dispersion.

In order to show the accuracy of the estimating results quantificationally, Figure 19 shows the root-mean-square values (Figure 19(a)) and mean values (Figure 19(b)) of errors of the five methods under 6 test cases. The computed results indicate that the present method and the Sadek-Simiu method can have a fair estimate of the positive and negative extreme values of the wind pressure.

\section{Conclusions}

The probabilistic and statistical characteristics of the wind pressure coefficients on a building surface and the disadvantages of the commonly used methods for their extreme values were discussed. Based on the GEV theory and the independence of the observed extreme values, the relationship of the extreme value distribution between the short-term sub-sample and the long-term maternal sample was established. A more accurate method of estimating extreme value was proposed. The following conclusions were obtained.

(1) Wind pressures on the surfaces of buildings, especially those on low-rise buildings exposed to 


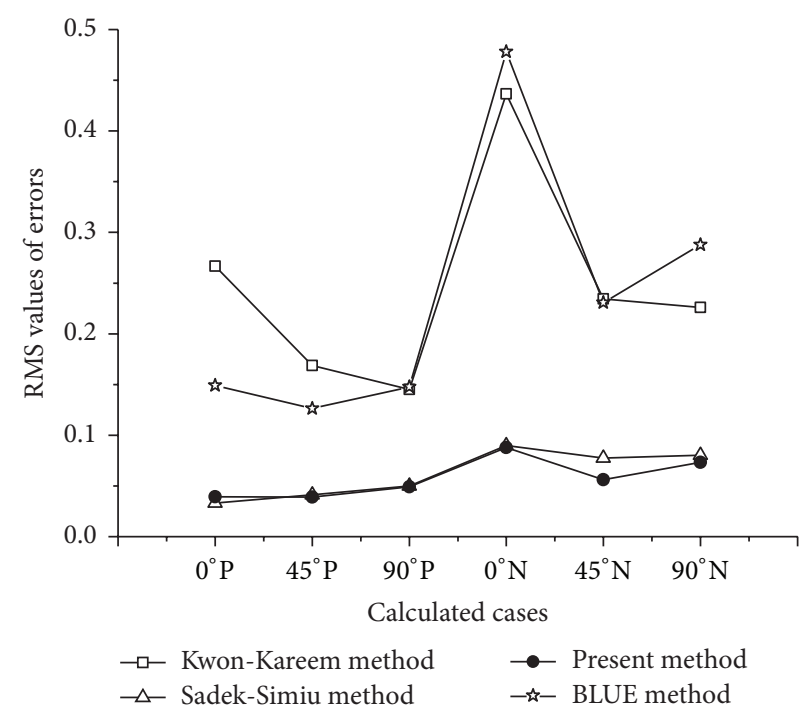

(a) Root-mean-square values of errors

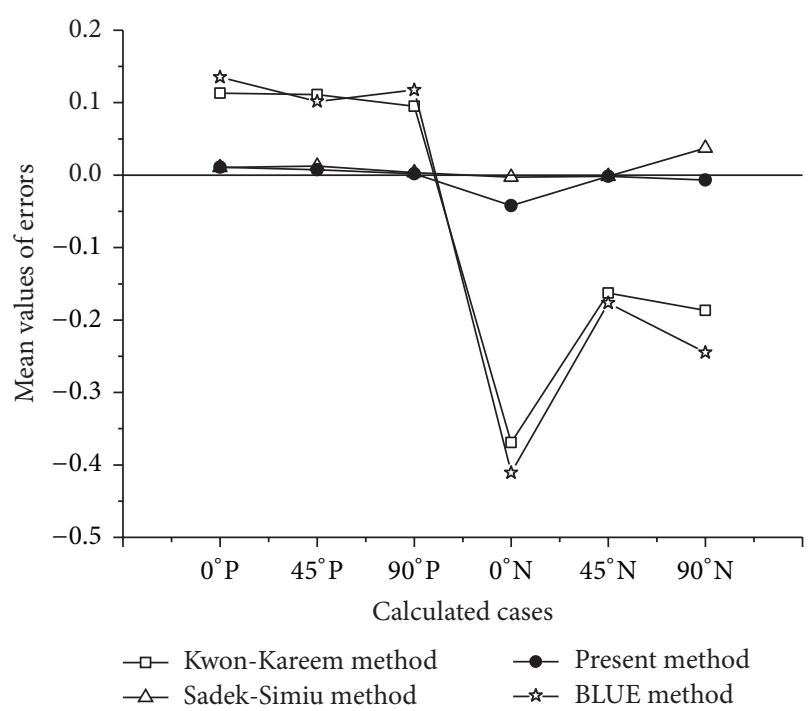

(b) Mean values of errors

FIGURE 19: Accuracy of the different methods under various working conditions of high-rise building (note that P represents the positive extreme values of calculated cases and $\mathrm{N}$ represents the negative extreme values of calculated cases).

high-turbulence air flow, do not follow a Gaussian distribution assumption and even seriously deviate from the Gaussian distribution in some cases.

(2) Whole wind pressures are difficult to fit to a single probability distribution function. Moreover, there is no consistency in the wind pressure behavior on the different locations.

(3) The extreme values of wind pressure coefficients on the high-rise building adhere to the extreme value types I and III. And the fitting results are not satisfactory enough in the rear part even if 2800 independent time series were taken. Although the number of independent extreme values from a single time history obtained from the sub-samples is far away from the requirement proposed by Holmes and Cochran [24], the GEV distribution function can describe the extreme values of the wind pressure coefficients more accurately than the extreme type I distribution.

(4) The Kwon-Kareem method attempts to reduce the error by introducing the non-Gaussian correction term. However, if the strong non-Gaussian process is encountered, the mapping result obtained by the polynomial established by the first four statistics would show a significant difference on both tails of the probability distribution. And when it was used to estimate the extreme values, the divergence of the results was significantly large.

(5) The conclusion drawn by Sadek and Simiu that the gamma and Gaussian distributions can describe the probability distribution of the wind pressure well is not as good as one thought. For those taps with strong non-Gaussianity, both tails of the probability distribution cannot be fitted satisfactorily by these two patterns, with great errors existing. According to the maximum domain of attraction theory, the gamma and Gaussian distribution both belong to the maximum attraction domain of extreme value type I distribution. And yet, the probability density distribution of the extreme values of wind pressure on the surfaces of buildings does not always comply with the extreme value type I. Consequently, it still leads to a relatively large error, especially when it was used to estimate the extreme values of wind pressures on the surfaces of low-rise building.

(6) The extreme values predicted by the BLUE method have significant errors sometimes because of the limitation of the extreme value type I distribution assumption.

(7) Compared with the commonly used methods, the present method can more accurately predict extreme values of positive and negative wind pressure coefficients.

\section{Conflict of Interests}

The authors declare that there is no conflict of interests regarding the publication of this paper.

\section{Acknowledgments}

The authors gratefully acknowledge the support from the National Natural Science Foundation of China (51278367, 90715040), the State Key Laboratory of Disaster Reduction in Civil Engineering in China (Grant no. SLDRCE10-B03), and the Fundamental Research Funds for the Central Universities. 


\section{References}

[1] A. G. Davenport, "The application of statistical concepts to the wind loading of structures," Proceedings of the Institution of Civil Engineers, vol. 19, no. 4, pp. 449-472, 1961.

[2] J. A. Peterka and J. E. Cermak, "Wind pressures on buildingsprobability densities," ASCE Journal of the Structural Division, vol. 101, no. 6, pp. 1255-1267, 1975.

[3] A. Kareem, Wind excited motion of tall buildings [Ph.D. dissertation in partial fulfilment of the degree of doctor of philosophy], Colorado State University, Fort Collins, Colo, USA, 1978.

[4] T. Stathopoulos, "PDF of wind pressures on low-rise buildings," ASCE Journal of the Structural Division, vol. 106, no. 5, pp. 973990, 1980.

[5] H. W. Tieleman and T. A. Reinhold, "Wind tunnel model investigation for basic dwelling geometrics," Tech. Rep. VPIE -76-8, Virginia Polytechnic Institute and State University, Richmond, Va, USA, 1976.

[6] J. D. Holmes and R. J. Best, "Wind pressures on an isolated highset house," Wind Engineering Report 1-78, Department of Civil Engineering, James Cook University of North Queensland, Townsville, Australia, 1978.

[7] J. D. Holmes, "Non-gaussian characteristics of wind pressure fluctuations," Journal of Wind Engineering and Industrial Aerodynamics, vol. 7, no. 1, pp. 103-108, 1981.

[8] H. Kawai, "Pressure fluctuations on square prisms-applicability of strip and quasi-steady theories," Journal of Wind Engineering and Industrial Aerodynamics, vol. 13, no. 1-3, pp. 197-208, 1983.

[9] C. W. Letchford, R. E. Iverson, and J. R. McDonald, “The application of the Quasi-steady Theory to full scale measurements on the Texas Tech building," Journal of Wind Engineering and Industrial Aerodynamics, vol. 48, no. 1, pp. 111-132, 1993.

[10] G. Thomas, P. P. Sarkar, and K. C. Mehta, "Identification of admittance functions for wind pressures from full-scale measurements," in Proceedings of the 9th International Conference on Wind Engineering, pp. 1219-1230, New Delhi, India, January 1995.

[11] H. W. Tieleman, M. R. Hajj, and T. A. Reinhold, "Pressure characteristics for separated flows," in Proceedings of the 10th International Conference on Wind Engineering, pp. 21-24, Copenhagen, Denmark, 1999.

[12] A. G. Davenport, "Note on the distribution of the largest value of arandom function with application to gust loading," Proceedings of the Institution of Civil Engineers, vol. 28, Paper no. 6739, no. 2, pp. 187-196.

[13] J. D. Holmes, "Wind action on glass and Brown's integral," Engineering Structures, vol. 7, no. 4, pp. 226-230, 1985.

[14] M. Gioffrè and V. Gusella, "Damage accumulation in glass plates," ASCE Journal of Engineering Mechanics, vol. 128, no. 7, pp. 801-805, 2002.

[15] A. Kareem and J. Zhao, "Analysis of non-gaussian surge response of tension leg platforms under wind loads," ASME Journal of Offshore Mechanics and Arctic Engineering, vol. 116, no. 3, pp. 137-144, 1994.

[16] D. E. Cartwright and M. S. Longuet-Higgins, "The Statistical distribution of the maxima of a random function," Proceedings of the Royal Society A, vol. 237, no. 1209, pp. 212-232, 1956.

[17] S. N. Pillai and Y. Tamura, "Generalized peak factor and its application to stationary random processes in wind engineering applications," Journal of Wind and Engineering, vol. 6, no. 1, supplement 1, pp. 1-10, 2009.
[18] D. Kwon and A. Kareem, "Peak factor for non-Gaussian processes revisited," in Proceedings of the 7th Asia-Pacific Conference on Wind Engineering, pp. 719-722, Taipei, Taiwan, November 2009.

[19] F. Sadek and E. Simiu, "Peak non-Gaussian wind effects for database-assisted low-rise building design," ASCE Journal of Engineering Mechanics, vol. 128, no. 5, pp. 530-539, 2002.

[20] M. Grigoriu, Applied Non-Gaussian Processes, Prentice Hall, Englewood Cliffs, NJ, USA, 1995.

[21] Z. Ge, Analysis of surface pressure and velocity fluctuations in the flow over surface-mounted prisms [Ph.D. dissertation], Department of Engineering Science and Mechanics, Virginia Polytechnic Institute and State University, Richmond, Va, USA, 2004.

[22] M. Kasperski, Specification and codification of design wind loads [Habilitation thesis], Department of Civil Engineering, Ruhr University Bochum, Bochum, Germany, 2000.

[23] J. D. Holmes and L. S. Cochran, "Probability distributions of extreme pressure coefficients," Journal of Wind Engineering and Industrial Aerodynamics, vol. 91, no. 7, pp. 893-901, 2003.

[24] J. D. Holmes and L. S. Cochran, "Distribution of extreme pressures," in Proceedings of the Americas Conference on Wind Engineering, pp. 4-6, Clemson, SC, USA, June 2001.

[25] J. A. Peterka, "Selection of local peak pressure coefficients for wind tunnel studies of buildings", Journal of Wind Engineering and Industrial Aerodynamics, vol. 13, no. 1-3, pp. 477-488, 1983.

[26] J. Lieblein, "Efficient methods of extreme-value methodology," Tech. Rep. 7507, National Bureau of Standards, New York, NY, USA, 1974.

[27] K. Kumar and T. Stathopoulos, "Wind loads on low building roofs: a stochastic perspective," Journal of Structural Engineering, vol. 126, no. 8, pp. 944-956, 2000.

[28] Y. F. Chen, S. B. Xu, Z. G. Sha, P. V. Gelder, and S. H. Gu, "Study on L-moment estimations for log-normal distribution with historical flood data," in GIS and Remote Sensing in Hydrology, Water Resources and Environment, vol. 289, pp. 107-113, International Association of Hydrological Sciences, Oxfordshire, UK, 2004.

[29] H. W. Tieleman and M. R. Hajj, "Theoretically estimated peak wind loads," in Proceedings of the 5th International Colloquium on Bluff Body Aerodynamics and Applications, pp. 359-362, Ottawa, Canada, July 2004.

[30] B. Chen, Research on the probabilistic and statistical characteristics of wind pressure on the roof of buildings and the estimating method of its extreme values [Master dissertation], Tongji University, Shanghai, China, 2008.

[31] R. A. Fisher and L. H. Tippett C, "Limiting forms of the frequency distribution of the largest or smallest members of a sample," Mathematical Proceedings of the Cambridge Philosophical Society, vol. 24, no. 2, pp. 180-190, 1928. 


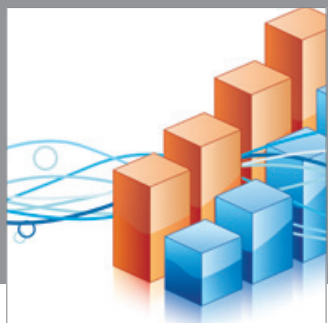

Advances in

Operations Research

mansans

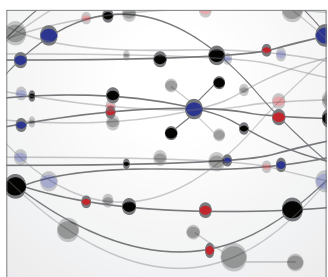

The Scientific World Journal
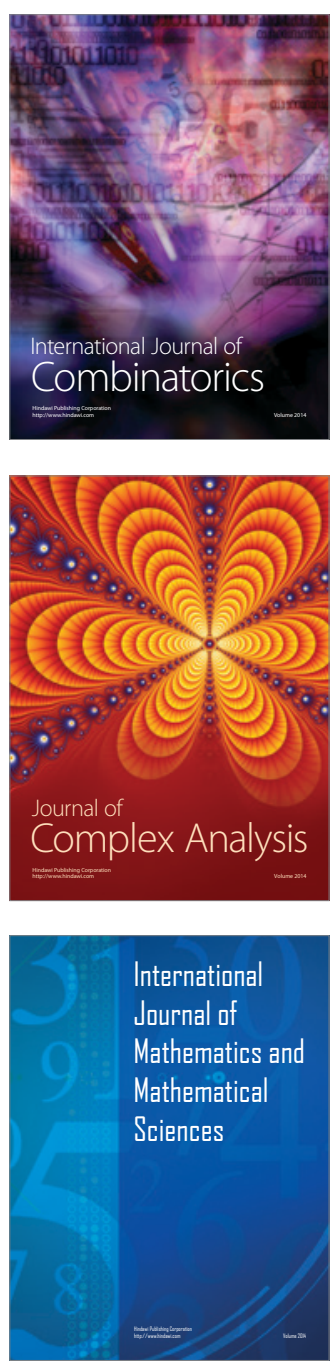
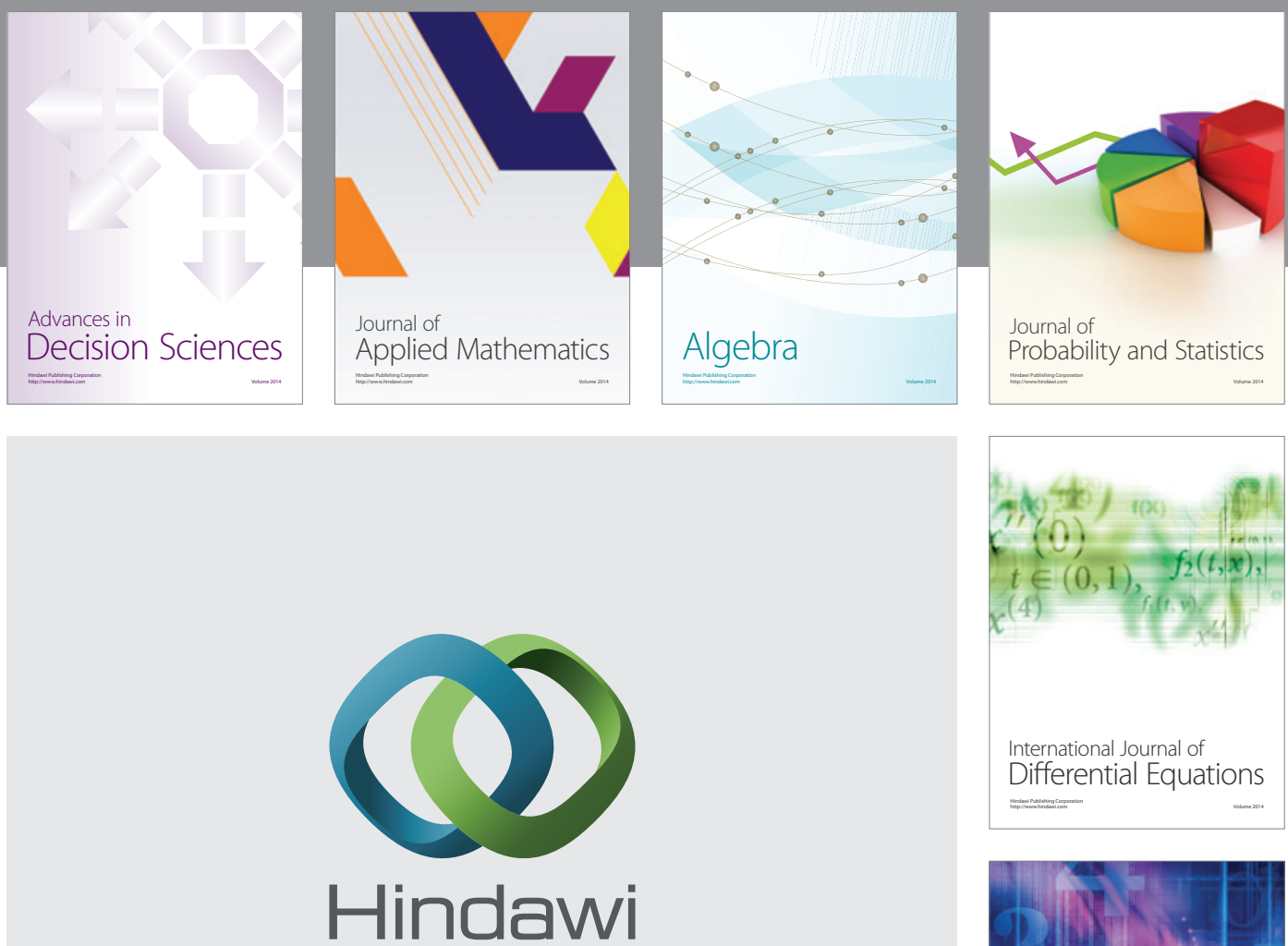

Submit your manuscripts at http://www.hindawi.com
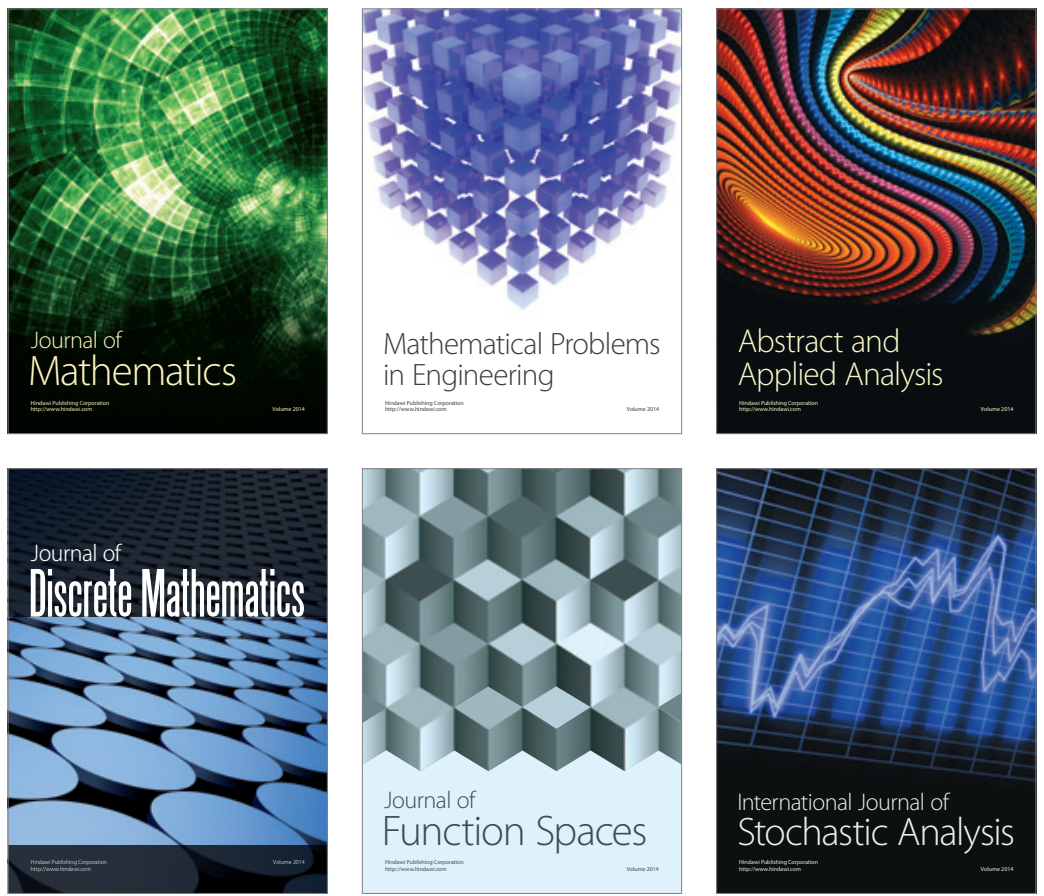

Journal of

Function Spaces

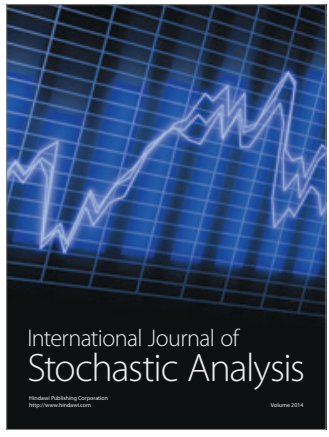

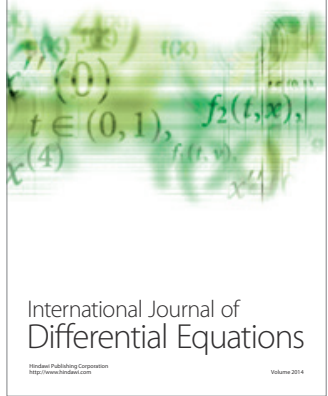
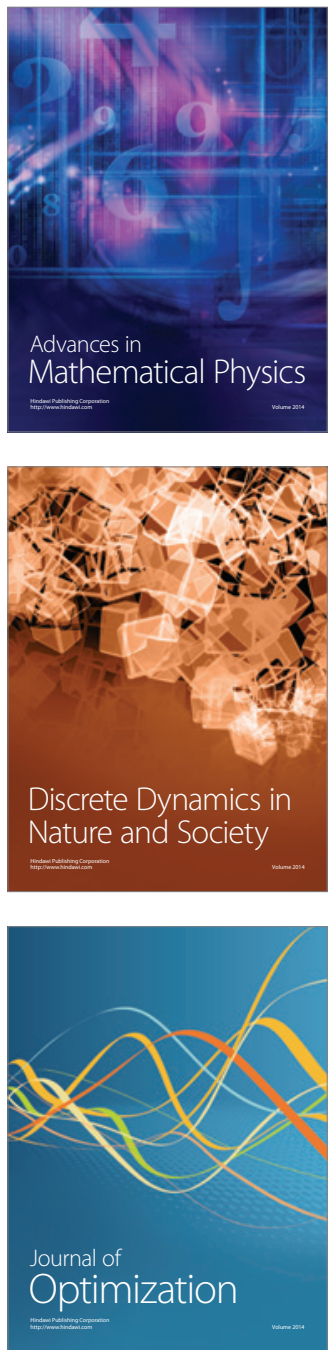